\title{
Topological sensitivity derivative with respect to area, shape and orientation of an elliptic hole in a plate
}

\author{
Dariusz Bojczuk • Zenon Mróz
}

Received: 5 January 2011 / Revised: 17 June 2011 / Accepted: 19 August 2011 / Published online: 27 September 2011

(C) The Author(s) 2011. This article is published with open access at Springerlink.com

\begin{abstract}
The topological sensitivity derivative of a functional expressed in terms of displacement, strain or stress fields and boundary tractions is derived for the case of an elliptical hole introduced in the plate. The derivative is specified with respect to the hole area, the length of ellipse axes and their orientation in terms of primary and adjoint state fields. The shape sensitivity derivative for a finite hole can be applied and the topological derivative with respect to the hole area is obtained in the limiting case. The transition to a plane crack occurs for vanishing length of minor axis and the topological derivative with respect to crack length is then derived from the general formulae. The results can be useful in optimal design procedures by selecting positions, shape and orientation of elliptical cutouts.
\end{abstract}

Keywords Topological sensitivity · Elliptical hole · Plate · Plane crack · Optimal design

\section{Introduction}

The present paper is aimed at obtaining the analytical form of the topological sensitivity derivative of an arbitrary strain and displacement functional or stress and boundary traction functional with respect to the area of elliptic hole introduced

D. Bojczuk (凶)

Faculty of Management and Computer Modelling, Kielce University of Technology, Kielce, Poland e-mail: mecdb@eden.tu.kielce.pl

\section{Z. Mróz}

Institute of Fundamental Technological Research, Polish Academy of Sciences, Warsaw, Poland

e-mail: zmroz@ippt.gov.pl in the plate subjected to boundary tractions and displacements. For an infinitesimal hole the derivative has finite value and can be applied in optimal design procedures by introducing elliptical holes, whose shape and orientation are obtained from the optimality conditions. The analytical form of the derivative is obtained by considering size variation of the elliptical hole of fixed shape and orientation of principal axes. Next, the sensitivity derivatives with respect to length of principal axes and their orientation are analytically expressed in closed form, not available in literature. The present derivation generalizes the previous works of Sokołowski and Żochowski (1999, 2003), who obtained the sensitivity derivative for a circular hole. The general formulae for topological derivatives in linear elasticity for arbitrary shapes of holes or inclusions have been derived by the method of compound asymptotic expansions with the use of polarization tensors by Nazarov and Sokołowski (2003) and Nazarov et al. (2010). Similarly, Amstutz (2006) presented the sensitivity analysis with respect to inhomogeneity perturbation for a class of problems described by Helmholtz type state equation, with the sensitivity expressed in terms of the polarization matrix. The inverse problems of inhomogeneity reconstruction using topological derivative concept were discussed by Ammari and Kang (2004).

The topological sensitivity analysis related to crack nucleation and growth was applied in several papers by Van Goethem and Novotny (2010), Feijóo et al. (2000), Khludnev et al. (2009), Novotny et al. (2003), Silva et al. (2010, 2011). Taroco (2000) derived first and second order shape sensitivity derivatives of the potential energy expressed in terms of path independent integrals and next related to crack growth. Kienzler et al. (2006) analyzed energy changes due to formation of a circular hole in a plate and presented several methods of specification of the potential energy variation including the application of the path 
independent integral related to growth of inhomogeneity. The class of conservation rules associated with the sensitivity analysis for translation, rotation and expansion of inhomogeneity was derived by Dems and Mróz (1986) and applied to crack sensitivity analysis.

The shape and topological size or area sensitivity derivatives are essential elements in topology optimization of structural elements using homogenization, variable density approaches, or level set methods etc., cf. Cea et al. (2000), Burger et al. (2004), Allaire et al. (2004), Wang et al. (2004), Bendsoe and Sigmund (2003), Bojczuk and Szteleblak (2008). The extensive review of topology optimization of structures was presented by Eschenauer and Olhoff (2001) and recently by Rozvany (2009). They are also important in material science, when the microstructure evolution corresponds to generation and growth of voids, inclusions, cracks or different crystalline phases, cf. Mróz and Bojczuk (2006).

The paper is organized as follows. In Section 2 the topological sensitivy derivative is expressed in terms of primary and adjoint stress states assuming the remote boundary loading to generate a uniform stress field in a plate with no hole inside its domain. The derivation follows the earlier works by Dems and Mróz (1984) and Bojczuk and Mróz (2009). In Section 3 the shape and orientation sensitivity analysis is presented. It is demonstrated that the topological derivative with respect to the hole area can be obtained from the shape sensitivity analysis assuming vanishing size parameter, as it was proposed by Novotny et al. (2003). The transition to the case of plane crack is obtained by setting the minor ellipse semiaxis length to tend to zero. The sensitivity derivatives with respect to the crack length are then generated and the stress intensity factors specified.

\section{Topological sensitivity derivative}

Consider now an elastic plate, whose middle surface occupies the domain $A \subset R^{2}$, with the boundary $\Gamma=\Gamma_{u} \cup \Gamma_{T}$. The plate is loaded by tractions $\mathbf{T}=\mathbf{T}^{0}\left(T_{i}^{0}=\sigma_{i j} n_{j}^{\Gamma}\right.$, $i, j=1,2)$ on the boundary portion $\Gamma_{T}$ and by body forces $\mathbf{p}^{0}$ in the plate domain $A$, where $\mathbf{n}^{\Gamma}=\left[n_{1}^{\Gamma}, n_{2}^{\Gamma}\right]$ is the unit vector normal to the boundary $\Gamma_{T}$ and $\sigma_{i j}$ represent components of the stress tensor. The equilibrium equations can be written in the form $\sigma_{i j, j}+p_{i}^{0}=0, i, j=1,2$. Moreover, displacements $\mathbf{u}=\mathbf{u}^{0}$ are specified on the boundary portion $\Gamma_{u}$.

The topological derivative of the functional $G$ with respect to the elliptical hole area is defined as follows (cf. Sokołowski and Żochowski 1999)

$T_{, A_{0}}^{G}(\mathbf{x}, \alpha)=\lim _{\xi \rightarrow 0} \frac{G\left(A_{\xi}\right)-G(A)}{\pi a b \xi^{2}}, \quad \mathrm{x} \in A$, where $\mathbf{x}$ is an arbitrary position in the plate domain, in which the derivative is specified, and $A_{\xi}=A-B_{\xi}(\mathbf{x}, \alpha)$. Here, $B_{\xi}(\mathbf{x}, \alpha)$ denotes the elliptical hole with its center at point $\mathbf{x}$, semi-axes $\xi a$ and $\xi b$, and the orientation angle $\alpha$ between the semi-axis $\xi a$ and the axis of principal stress $\sigma_{1}$, Fig. 1 .

The expansion parameter $\xi$ specifies the size of the elliptical hole. The coordinate axes $x_{1}, x_{2}$ follow the orientations of principal stresses $\sigma_{1}, \sigma_{2}$ specified in the plate before introduction of hole. It is assumed that the ratio $\eta=b / a$ specifying ellipse shape and the principal axes orientation angle $\alpha$ is fixed.

2.1 Sensitivity derivative for the displacement and strain functional with respect to expansion parameter $\xi$

Consider the functional in the form

$G=\int_{A_{\xi}} F(\varepsilon) d A_{\xi}+\int_{A_{\xi}} f(\mathbf{u}) d A_{\xi}+\int_{\Gamma_{T}} g(\mathbf{u}) d \Gamma_{T}$,

where $F$ is a function of strain vector $\varepsilon=\left[\varepsilon_{11}, \varepsilon_{22}, 2 \varepsilon_{12}\right]^{T}$, $f$ and $g$ are functions of displacements $\mathbf{u}$ and $A_{\xi}$ denotes the plate domain with the elliptical hole, whose size is defined by the parameter $\xi$. The integrand functions are assumed to be differentiable with respect to their arguments.

It can be demonstrated from the asymptotic analysis (cf. Gao 1996), that

$\left.\mathbf{u}\right|_{\xi=0}=\mathbf{u}^{(0)},\left.\varepsilon\right|_{\xi=0}=\varepsilon^{(0)}$ in $A$,

where $\left.\mathbf{u}\right|_{\xi=0}$ and $\left.\varepsilon\right|_{\xi=0}$ denote the displacement and strain states for the plate with elliptic hole for $\xi \rightarrow 0$, and $\mathbf{u}^{(0)}$,

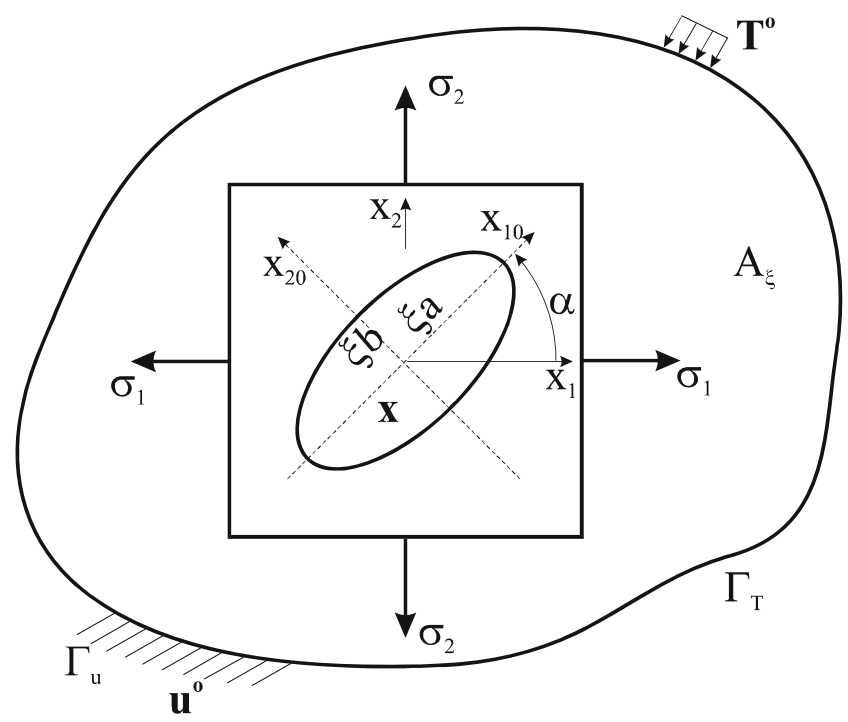

Fig. 1 Introduction of elliptical hole with semi-axes $\xi a$ and $\xi b$ 
$\mathcal{\varepsilon}^{(0)}$ are the states for a homogeneous plate. In view of (3) there is

$\lim _{\xi \rightarrow 0} G=G^{*}=G(A)$

where

$G^{*}=\int_{A} F\left(\varepsilon^{(0)}\right) d A+\int_{A} f\left(\mathbf{u}^{(0)}\right) d A+\int_{\Gamma_{T}} g\left(\mathbf{u}^{(0)}\right) d \Gamma_{T}$,

denotes a value of the functional for the homogeneous plate.

Thus, the continuity of functional (2) occurs for $\xi \rightarrow 0$ and the derivative (1) with respect to hole area for $\xi=0$ can be calculated assuming nucleation and growth of hole of fixed geometric and orientation parameters $a, b, \eta$ and $\alpha$.

The variational approach is applied to specify the sensitivity derivative, and the first variation of functional (2) in the hole expansion process at the material point $\mathbf{x}$ is expressed as follows

$$
\begin{aligned}
\delta G= & \int_{A_{\xi}} \frac{\partial F}{\partial \varepsilon} \cdot \delta \varepsilon d A_{\xi}+\int_{A_{\xi}} \frac{\partial f}{\partial \mathbf{u}} \cdot \delta \mathbf{u} d A_{\xi}+\int_{\Gamma_{T}} \frac{\partial g}{\partial \mathbf{u}} \cdot \delta \mathbf{u} d \Gamma_{T} \\
& +\sum_{k=1}^{2} \int_{\Gamma_{\xi}}(F+f) n_{k} \delta \Psi_{k} d \Gamma_{\xi}
\end{aligned}
$$

where $\mathbf{n}=\left[n_{1}, n_{2}\right]^{T}$ is the unit vector normal to the hole boundary $\Gamma_{\xi}, \delta \boldsymbol{\Psi}=\left[\delta \boldsymbol{\Psi}_{1}, \delta \boldsymbol{\Psi}_{2}\right]^{T}$ is the hole boundary incremental transformation vector and (.) denotes the scalar product. Following the previous derivation for plates cf. Bojczuk and Mróz (2009) and the general methodology of sensitivity analysis cf. Dems and Mróz (1984), the variations of state fields can be eliminated by introducing an adjoint plate structure of the same form, as the primary plate, but with induced initial stress and body force fields, namely

$\boldsymbol{\sigma}^{a i}=E \varepsilon^{a i}=\frac{\partial F}{\partial \varepsilon}$ in $A_{\xi}, \quad \mathbf{p}^{a 0}=\frac{\partial f}{\partial \mathbf{u}}$ in $A_{\xi}$

and satisfying the following boundary conditions

$\mathbf{T}^{a}=\mathbf{T}^{a 0}=\frac{\partial g}{\partial \mathbf{u}}$ on $\Gamma_{T}, \quad \mathbf{u}^{a}=\mathbf{u}^{a 0}=\mathbf{0}$ on $\Gamma_{u}$.

Here $\sigma^{a}, \mathbf{u}^{a}, \varepsilon^{a}$ are the state vector fields in the adjoint structure. The stress field $\sigma^{a}=\left[\sigma_{11}^{a}, \sigma_{22}^{a}, \sigma_{12}^{a}\right]^{T}$ is a following sum

$\sigma^{a}=\sigma^{a i}+\sigma^{a r}$ where $\sigma^{a r}=\sigma^{a}-\sigma^{a i}=E\left(\varepsilon^{a}-\varepsilon^{a i}\right)$ is the elastic stress field and then $T_{i}^{a 0}=\sigma_{i j}^{a r} n_{j}^{\Gamma}, i, j=1,2$. Now, the equilibrium equations can be written in the form $\sigma_{i j, j}^{a r}+p_{i}^{a 0}=$ $0, i, j=1,2$. In view of (7) and (8) the functional variation (6) can be rewritten as follows

$$
\begin{aligned}
\delta G= & \int_{A_{\xi}} \boldsymbol{\sigma}^{a i} \cdot \delta \mathcal{E} d A_{\xi}+\int_{A_{\xi}} \mathbf{p}^{a 0} \cdot \delta \mathbf{u} d A_{\xi}+\int_{\Gamma_{T}} \mathbf{T}^{a 0} \cdot \delta \mathbf{u} d \Gamma_{T} \\
& +\sum_{k=1}^{2} \int_{\Gamma_{\xi}}(F+f) n_{k} \delta \boldsymbol{\Psi}_{k} d \Gamma_{\xi} .
\end{aligned}
$$

The virtual work equation for the adjoint structure can be written in the form

$$
\int_{A_{\xi}} \boldsymbol{\sigma}^{a r} \cdot \delta \varepsilon d A_{\xi}=\int_{A_{\xi}} \mathbf{p}^{a 0} \cdot \delta \mathbf{u} d A_{\xi}+\int_{\Gamma_{T}} \mathbf{T}^{a 0} \cdot \delta \mathbf{u} d \Gamma_{T},
$$

since $\sigma^{a r}$ satisfies the equilibrium equations and boundary conditions of the adjoint structure and $\mathbf{u}(\mathbf{x}), \varepsilon(\mathbf{x})$ are the kinematically admissible displacement and strain fields.

The virtual work equation for the stress increment $\delta \sigma$ of the primary structure and the kinematically admissible displacement field of the adjoint structure $\mathbf{u}^{a}$ can be written as follows

$$
\begin{aligned}
\int_{A_{\xi}} \boldsymbol{\sigma}^{a} \cdot \delta \mathfrak{\varepsilon} d A_{\xi}= & \int_{A_{\xi}} \mathcal{\varepsilon}^{a} \cdot \delta \boldsymbol{\sigma} d A_{\xi}=-\sum_{k=1}^{2} \int_{\Gamma_{\xi}} \boldsymbol{\sigma} \cdot \varepsilon^{a} n_{k} \delta \boldsymbol{\Psi}_{k} d \Gamma_{\xi} \\
& +\sum_{k=1}^{2} \int_{\Gamma_{\xi}} \mathbf{p}^{0} \cdot \mathbf{u}^{a} n_{k} \delta \boldsymbol{\Psi}_{k} d \Gamma_{\xi} .
\end{aligned}
$$

Accounting for (9), (11) and (12), the following equality can be derived

$$
\begin{aligned}
\int_{A_{\xi}} \boldsymbol{\sigma}^{a i} \cdot \delta \mathcal{\varepsilon} d A_{\xi}= & \int_{A_{\xi}} \boldsymbol{\sigma}^{a} \cdot \delta \varepsilon d A_{\xi}-\int_{A_{\xi}} \boldsymbol{\sigma}^{a r} \cdot \delta \mathfrak{\varepsilon} d A_{\xi} \\
= & \sum_{k=1}^{2} \int_{\Gamma_{\xi}}\left(\mathbf{p}^{0} \cdot \mathbf{u}^{a}-\boldsymbol{\sigma} \cdot \boldsymbol{\varepsilon}^{a}\right) n_{k} \delta \boldsymbol{\Psi}_{k} d \Gamma_{\xi} \\
& -\int_{A_{\xi}} \mathbf{p}^{a 0} \cdot \delta \mathbf{u} d A_{\xi}-\int_{\Gamma_{T}} \mathbf{T}^{a 0} \cdot \delta \mathbf{u} d \Gamma_{T} .
\end{aligned}
$$


In view of (13), the expression for the functional variation can be derived in terms of primary, adjoint fields and normal transformation of the hole boundary $\Gamma_{\xi}$, namely

$\delta G=\sum_{k=1}^{2} \int_{\Gamma_{\xi}}\left(-\boldsymbol{\sigma} \cdot \varepsilon^{a}+\mathbf{p}^{0} \cdot \mathbf{u}^{a}+F+f\right) n_{k} \delta \boldsymbol{\Psi}_{k} d \Gamma_{\xi}$.

Introduce the local coordinate system $x_{10}, x_{20}$ with axes coinciding with elliptic hole semi-axes $\xi a$ and $\xi b$, Fig. 2. The coordinates of boundary points and their increments are

$$
x_{10}=\xi a \cos \theta, \quad x_{20}=\xi b \sin \theta,
$$

$d x_{10}=-\xi a \sin \theta d \theta, \quad d x_{20}=\xi b \cos \theta d \theta$.

The unit normal vector to the boundary $\Gamma_{\xi}$ can be expressed as follows

$$
\begin{aligned}
\mathbf{n} & =\left[-\frac{d x_{20}}{\sqrt{d x_{10}^{2}+d x_{20}^{2}}}, \frac{d x_{10}}{\sqrt{d x_{10}^{2}+d x_{20}^{2}}}\right]^{T} \\
& =\left[-\frac{b \cos \theta}{\sqrt{a^{2} \sin ^{2} \theta+b^{2} \cos ^{2} \theta}},-\frac{a \sin \theta}{\sqrt{a^{2} \sin ^{2} \theta+b^{2} \cos ^{2} \theta}}\right]^{T}
\end{aligned}
$$

and the length element of the hole perimeter is

$$
d \Gamma_{\xi}=\sqrt{d x_{10}^{2}+d x_{20}^{2}}=\xi \sqrt{a^{2} \sin ^{2} \theta+b^{2} \cos ^{2} \theta} d \theta .
$$

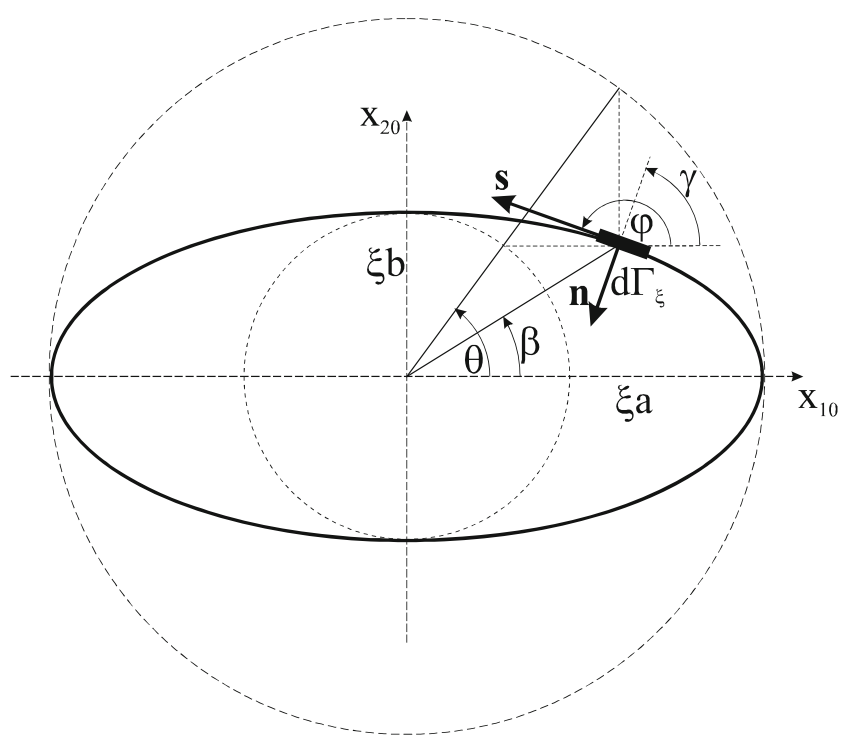

Fig. 2 Elliptical hole: local coordinates and notations
The transformation function specifying the radial boundary evolution now takes the form

$\boldsymbol{\Psi}=\left[\begin{array}{ll}\xi a \cos \theta, & \xi b \sin \theta\end{array}\right]^{T}$

and its variation is

$\delta \Psi=\frac{\partial \Psi}{\partial \xi} \delta \xi=[a \cos \theta \delta \xi, \quad b \sin \theta \delta \xi]^{T}$.

So, in view of (16), (17) and (19), the variation of the functional $G$ expressed by (14), can be presented in the form

$$
\begin{aligned}
\delta G= & \int_{0}^{2 \pi}\left(-\sigma \cdot \varepsilon^{a}+\mathbf{p}^{0} \cdot \mathbf{u}^{a}+F+f\right) \\
& \times\left(-\frac{a b \cos ^{2} \theta+a b \sin ^{2} \theta}{\sqrt{a^{2} \sin ^{2} \theta+b^{2} \cos ^{2} \theta}} \delta \xi\right) \\
& \times \xi \sqrt{a^{2} \sin ^{2} \theta+b^{2} \cos ^{2} \theta} d \theta \\
= & \xi a b \int_{0}^{2 \pi}\left(\sigma \cdot \varepsilon^{a}-\mathbf{p}^{0} \cdot \mathbf{u}^{a}-F-f\right) d \theta \delta \xi .
\end{aligned}
$$

The sensitivity derivative of $G$ with respect to size parameter is

$$
\frac{\partial G}{\partial \xi}=\xi a b \int_{0}^{2 \pi}\left(\sigma \cdot \varepsilon^{a}-\mathbf{p}^{0} \cdot \mathbf{u}^{a}-F-f\right) d \theta
$$

and it vanishes for $\xi=0$. To express sensitivity derivative with respect to the hole area $A=\pi \xi^{2} a b$, the following formula is obtained using the relation (21) and the incremental form $d A=2 \pi \xi a b d \xi$, thus (cf. Novotny et al. 2003)

$$
\begin{aligned}
T_{, A_{0}}^{G}(\mathbf{x}, \alpha)= & \left.\left(\frac{\partial G}{\partial \xi} \frac{\partial \xi}{\partial A}\right)\right|_{\xi=0} \\
& =\frac{1}{2 \pi} \int_{0}^{2 \pi}\left(\sigma \cdot \varepsilon^{a}-\mathbf{p}^{0} \cdot \mathbf{u}^{a}-F-f\right) d \theta .
\end{aligned}
$$

To express integrand of (22) in terms of the local stress components, introduce the coordinate system $s, n$ by the unit tangent and normal vectors to the hole boundary, Fig. 2 . Then on the boundary $\Gamma_{\xi}$, we have

$\sigma_{n n}=\sigma_{s n}=0, \quad \sigma_{n n}^{a}=\sigma_{s n}^{a}=0$,

and the non-vanishing terms are $\sigma_{s s}$ and $\sigma_{s s}^{a}$. We then get

$\sigma \cdot \varepsilon^{a}=\sigma_{s s} \varepsilon_{s s}^{a}=\frac{1}{E} \sigma_{s s} \sigma_{s s}^{a}$, 
where $\varepsilon_{s s}^{a}=(1 / E) \sigma_{s s}^{a}$ and $E$ denotes Young modulus. Further, assume the function $F(\varepsilon)$ in the form

$F(\varepsilon)=\left(\varepsilon^{T} \mathbf{S} \varepsilon\right)^{p}$

where the matrix $\mathbf{S}$ is assumed as follows

$\mathbf{S}=\left[\begin{array}{ccc}l+2 m & l & 0 \\ l & l+2 m & 0 \\ 0 & 0 & m\end{array}\right]$

and $p, l, m$ are the selected parameters. In particular, the parameters $l$ and $m$ can correspond to elastic moduli $\lambda, \mu$ of the linear isotropic material. Referring the local stress state $\sigma_{s s}\left(\sigma_{n n}=\sigma_{s n}=0\right)$ to the reference system $x_{10}, x_{20}$ coinciding with the elliptic hole semi-axes at $\mathbf{x}$ from Hooke's law, at first we have

$\varepsilon_{11}=\frac{1}{E}\left(\sigma_{11}-v \sigma_{22}\right), \quad \varepsilon_{11}=\frac{1}{E}\left(\sigma_{11}-v \sigma_{22}\right)$,

$\gamma_{12}=\frac{\sigma_{12}}{G}$

where $v$ is the Poisson's ratio and $G=E /[2(1+v)]$ denotes the Kirchhoff modulus. From the transformation formulae for the stress state on the hole boundary, we get

$\sigma_{11}=\frac{1}{2} \sigma_{s s}(1+\cos 2 \varphi), \quad \sigma_{22}=\frac{1}{2} \sigma_{s s}(1-\cos 2 \varphi)$,

$\sigma_{12}=\frac{1}{2} \sigma_{s s} \sin 2 \varphi$

where $\varphi$ is the angle between $x_{10}$ and $s$ axes (Fig. 2). Now, the function $F$ can be expressed in terms of stress component $\sigma_{s S}$ as follows

$F\left(\sigma_{s s}\right)=\left\{\left[\left(1+v^{2}\right)(l+2 m)-2 v l\right] \frac{\sigma_{s s}^{2}}{E^{2}}\right\}^{p}$.

In view of the equality

$\int_{0}^{2 \pi}\left[\mathbf{p}^{0} \cdot \mathbf{u}^{a}+f(\mathbf{u})\right] d \theta=2 \pi\left[\mathbf{p}^{0} \cdot \mathbf{u}^{a}+f(\mathbf{u})\right]$,

formulae (24), (29) and assuming $p=1$, the sensitivity derivative can be expressed as follows

$$
\begin{aligned}
T_{, A_{0}}^{G}(\mathbf{x}, \alpha)= & \frac{1}{2 \pi E} \int_{0}^{2 \pi} \sigma_{s s} \sigma_{s s}^{a} d \theta \\
& -\frac{1}{2 \pi} \int_{0}^{2 \pi}\left[\left(1+v^{2}\right)(l+2 m)-2 v l\right] \frac{\sigma_{s s}^{2}}{E^{2}} d \theta \\
& -\left[\mathbf{p}^{0} \cdot \mathbf{u}^{a}+f(\mathbf{u})\right] .
\end{aligned}
$$

Consider now the elliptical hole in the primary plate with its major axis oriented at angle $\alpha$ to the principal stress $\sigma_{1}$ acting along the coordinate axis $x_{1}$, Fig. 3a. The tangential stress distribution at the hole perimeter is specified from the elastic solution for an infinite plate with an elliptic hole, subjected to biaxial loading, cf. Gao (1996) or Timoshenko and Goodier (1951), thus

$$
\begin{aligned}
\sigma_{s s}= & \left(\sigma_{1}+\sigma_{2}\right) \frac{1-M^{2}}{M^{2}-2 M \cos 2 \theta+1} \\
& +2\left(\sigma_{1}-\sigma_{2}\right) \frac{M \cos 2 \alpha-\cos 2(\theta+\alpha)}{M^{2}-2 M \cos 2 \theta+1},
\end{aligned}
$$

where $\sigma_{1}, \sigma_{2}$ are the principal stresses at the point $\mathbf{x}$ of the domain $A$ without the elliptical hole, the angle $\theta$ specifies the position on the perimeter with respect to the major ellipse axis, Fig. 2, and

$M=\frac{a-b}{a+b}=\frac{1-\eta}{1+\eta}, \quad \eta=\frac{b}{a}$.

Fig. 3 The elliptical hole: a) in the primary plate; $\mathbf{b}$ ) in the adjoint plate, with reference to principal stress axes a)

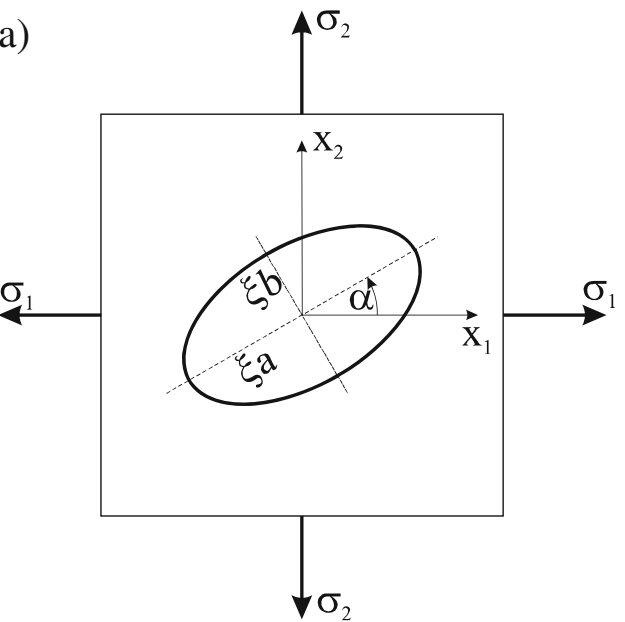

b)

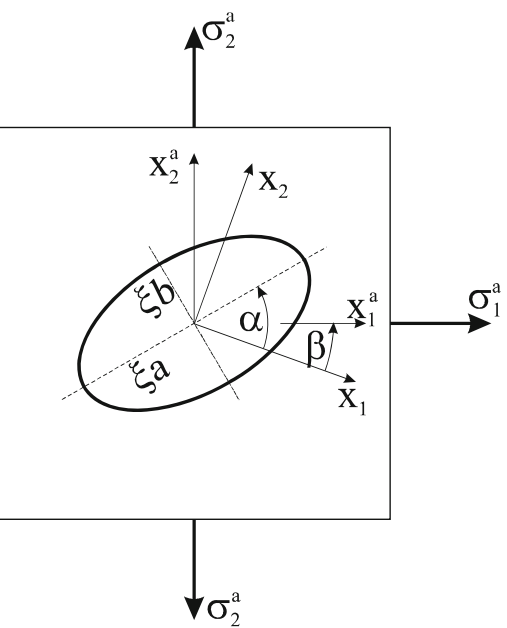


The alternative form of (32) in terms of the shape parameter $\eta$ is

$$
\begin{aligned}
\sigma_{s s}= & \left(\sigma_{1}+\sigma_{2}\right) \frac{2 \eta}{1+\eta^{2}-\left(1-\eta^{2}\right) \cos 2 \theta} \\
& +\left(\sigma_{1}-\sigma_{2}\right) \frac{\left(1-\eta^{2}\right) \cos 2 \alpha-(1+\eta)^{2} \cos 2(\theta+\alpha)}{1+\eta^{2}-\left(1-\eta^{2}\right) \cos 2 \theta} .
\end{aligned}
$$

It is important to notice that determination of $\sigma_{s s}$ from the solution for an infinite plate under uniform stress state is an approximation and range of its validity was discussed by Silva et al. (2010). However, the expression (31) is still presented in general form and can be applied to cases of non-uniform stress states in structural elements.

Next, consider the adjoint structure, where the principal stress $\sigma_{1}^{a}$ acting along the coordinate axis $x_{1}^{a}$ is oriented at the angle $\beta$ to the $x_{1}$ axis and inclined at the angle $\alpha-\beta$ to the major ellipse axis, Fig. 3b. Now, the distribution of the adjoint stress $\sigma_{s s}^{a}$ on the elliptic hole boundary is expressed similarly as in the primary structure, namely

$$
\begin{aligned}
\sigma_{s s}^{a}= & \left(\sigma_{1}^{a}+\sigma_{2}^{a}\right) \frac{1-M^{2}}{M^{2}-2 M \cos 2 \theta+1} \\
& +2\left(\sigma_{1}^{a}-\sigma_{2}^{a}\right) \frac{M \cos 2(\alpha-\beta)-\cos 2(\theta+\alpha-\beta)}{M^{2}-2 M \cos 2 \theta+1},
\end{aligned}
$$

or

$$
\begin{aligned}
\sigma_{s s}^{a}= & \left(\sigma_{1}^{a}+\sigma_{2}^{a}\right) \frac{2 \eta}{1+\eta^{2}-\left(1-\eta^{2}\right) \cos 2 \theta}+\left(\sigma_{1}^{a}-\sigma_{2}^{a}\right) \\
& \times \frac{\left(1-\eta^{2}\right) \cos 2(\alpha-\beta)-(1+\eta)^{2} \cos 2(\theta+\alpha-\beta)}{1+\eta^{2}-\left(1-\eta^{2}\right) \cos 2 \theta} .
\end{aligned}
$$

The integrals occurring in (31) can be presented in the form

$$
\begin{aligned}
\int_{0}^{2 \pi} \sigma_{s s} \sigma_{s s}^{a} d \theta= & \pi\left(\sigma_{1}+\sigma_{2}\right)\left(\sigma_{1}^{a}+\sigma_{2}^{a}\right)\left(\eta+\frac{1}{\eta}\right) \\
& +\pi\left(\sigma_{1}-\sigma_{2}\right)\left(\sigma_{1}^{a}+\sigma_{2}^{a}\right)\left(\eta-\frac{1}{\eta}\right) \\
& \times \cos 2 \alpha+\pi\left(\sigma_{1}+\sigma_{2}\right)\left(\sigma_{1}^{a}-\sigma_{2}^{a}\right)\left(\eta-\frac{1}{\eta}\right) \\
& \times \cos 2(\alpha-\beta)+\pi\left(\sigma_{1}-\sigma_{2}\right) \\
& \times\left(\sigma_{1}^{a}-\sigma_{2}^{a}\right)\left(\eta+2+\frac{1}{\eta}\right) \cos 2 \beta
\end{aligned}
$$

and

$$
\begin{aligned}
\int_{0}^{2 \pi} \sigma_{s s}^{2} d \theta= & \pi\left(\sigma_{1}+\sigma_{2}\right)^{2}\left(\eta+\frac{1}{\eta}\right)+2 \pi\left(\sigma_{1}^{2}-\sigma_{2}^{2}\right) \\
& \times\left(\eta-\frac{1}{\eta}\right) \cos 2 \alpha+\pi\left(\sigma_{1}-\sigma_{2}\right)^{2}\left(\eta+2+\frac{1}{\eta}\right) .
\end{aligned}
$$

Accounting for (37), (38), the topological sensitivity derivative (31) can be expressed analytically as follows

$$
\begin{aligned}
T_{, A_{0}}^{G}(\mathbf{x}, \alpha)=\frac{1}{2 E}[ & \left(\sigma_{1}+\sigma_{2}\right)\left(\sigma_{1}^{a}+\sigma_{2}^{a}\right)\left(\eta+\frac{1}{\eta}\right) \\
& +\left(\sigma_{1}-\sigma_{2}\right)\left(\sigma_{1}^{a}+\sigma_{2}^{a}\right)\left(\eta-\frac{1}{\eta}\right) \cos 2 \alpha \\
& +\left(\sigma_{1}+\sigma_{2}\right)\left(\sigma_{1}^{a}-\sigma_{2}^{a}\right)\left(\eta-\frac{1}{\eta}\right) \\
& \times \cos 2(\alpha-\beta)+\left(\sigma_{1}-\sigma_{2}\right) \\
& \left.\times\left(\sigma_{1}^{a}-\sigma_{2}^{a}\right)\left(\eta+2+\frac{1}{\eta}\right) \cos 2 \beta\right] \\
-\frac{\left(1+v^{2}\right)(l+2 m)-2 v l}{2 E^{2}} & \\
\times & \left(\sigma_{1}+\sigma_{2}\right)^{2}\left(\eta+\frac{1}{\eta}\right)+2\left(\sigma_{1}^{2}-\sigma_{2}^{2}\right) \\
& \times\left(\eta-\frac{1}{\eta}\right) \cos 2 \alpha+\left(\sigma_{1}-\sigma_{2}\right)^{2} \\
& \left.\times\left(\eta+2+\frac{1}{\eta}\right)\right]-\left[\mathbf{p}^{0} \cdot \mathbf{u}^{a}+f(\mathbf{u})\right] .
\end{aligned}
$$

The considered here case $p=1$ corresponds to the quadratic strain (or stress) integral function (25). The cases $p \neq 1$ can be treated numerically.

\subsection{Sensitivity derivative for the strain energy functional}

The particular case occurs when $f(\mathbf{u})=0$ on $\Gamma_{T}, g(\mathbf{u})$ in $A$, $\mathbf{p}^{0}=\mathbf{0}$ at the point $\mathbf{x}$ and the integrand function of (2) is the strain energy function $F(\varepsilon)=U(\varepsilon)$. This case corresponds to the strain or complementary energy variations. The selfadjoint sensitivity problem now is obtained and then

$\sigma_{s s}=\sigma_{s s}^{a}, \quad l=\frac{v E}{2\left(1-v^{2}\right)}, \quad m=\frac{E}{4(1+v)}=\frac{G}{2}$. 
The topological sensitivity derivative then equals

$$
\begin{aligned}
T_{, A_{0}}^{U}(\mathbf{x}, \alpha)=\frac{1}{4 E}[ & \left(\sigma_{1}+\sigma_{2}\right)^{2}\left(\eta+\frac{1}{\eta}\right) \\
& +2\left(\sigma_{1}^{2}-\sigma_{2}^{2}\right)\left(\eta-\frac{1}{\eta}\right) \cos 2 \alpha \\
& \left.+\left(\sigma_{1}-\sigma_{2}\right)^{2}\left(\eta+2+\frac{1}{\eta}\right)\right] .
\end{aligned}
$$

The diagrams of topological derivative variation of the strain energy at arbitrary point $\mathbf{x}$ of the infinite plate in biaxial uniform stress state, in function of shape parameters $\eta=$ $b / a, 1 / \eta=a / b$, for different values of stress ratio $\zeta=$ $\sigma_{2} / \sigma_{1}$ and orientation angle $\alpha$, are presented in Fig. 4. Let us note, that in the case $\alpha=0$ and for $\zeta \in(0 ; 1\rangle$, topological derivative attains minimum for the shape parameter $\eta=\zeta$. In the case $\alpha=\pi / 4$ the minimum always occurs for $\eta=1$.

The topological derivative can also be expressed in terms of components $\sigma_{11}, \sigma_{22}, \sigma_{12}$ of the stress field in the coordi- nates $x_{10}, x_{20}$ located along ellipse axes. In this case, using the stress transformation formulae, we have

$$
\begin{aligned}
T_{, A_{0}}^{U}(\mathbf{x})= & \frac{1}{4 E}\left\{\left(\sigma_{11}+\sigma_{22}\right)^{2}\left(\eta+\frac{1}{\eta}\right)+2\left(\sigma_{11}^{2}-\sigma_{22}^{2}\right)\right. \\
& \times\left(\eta-\frac{1}{\eta}\right)+\left[\left(\sigma_{11}-\sigma_{22}\right)^{2}+4 \sigma_{12}^{2}\right] \\
& \left.\times\left(\eta+2+\frac{1}{\eta}\right)\right\}
\end{aligned}
$$

2.3 Sensitivity derivative for the stress and reaction traction functional

Now, let us consider the following functional of stress state and boundary tractions on $\Gamma_{u}$

$G=\int_{A_{\xi}} H(\boldsymbol{\sigma}) d A_{\xi}+\int_{\Gamma_{u}} h(\mathbf{T}) d \Gamma_{u}$,

where $H(\sigma)$ is the function of stress state $\sigma=\left[\sigma_{11}\right.$, $\left.\sigma_{22}, \sigma_{12}\right]^{T}$ and $h(\mathbf{T})$ denotes function of reaction tractions
Fig. 4 Non-dimensional topological derivative variation of the strain energy $\frac{E}{\sigma_{1}^{2}} \cdot T_{, A_{0}}^{U}$ in function of shape parameters $\eta=b / a, 1 / \eta=a / b$, for different values of stress ratio $\zeta=\sigma_{2} / \sigma_{1}$ and for orientation angles: a) $\alpha=0$; b) $\alpha=\pi / 4$ a)

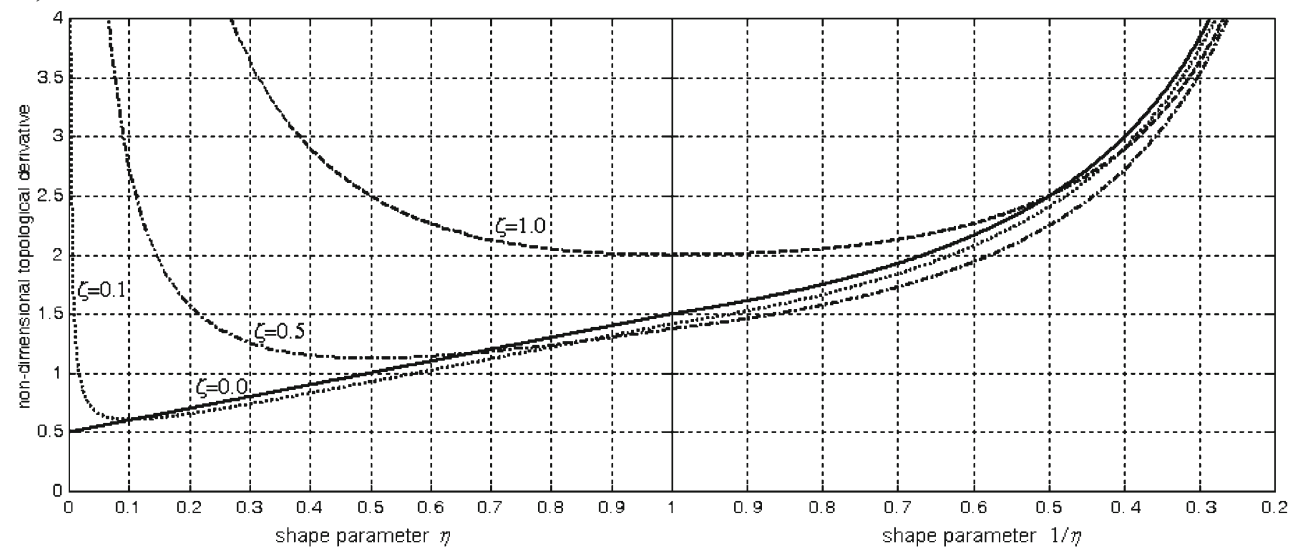

b)

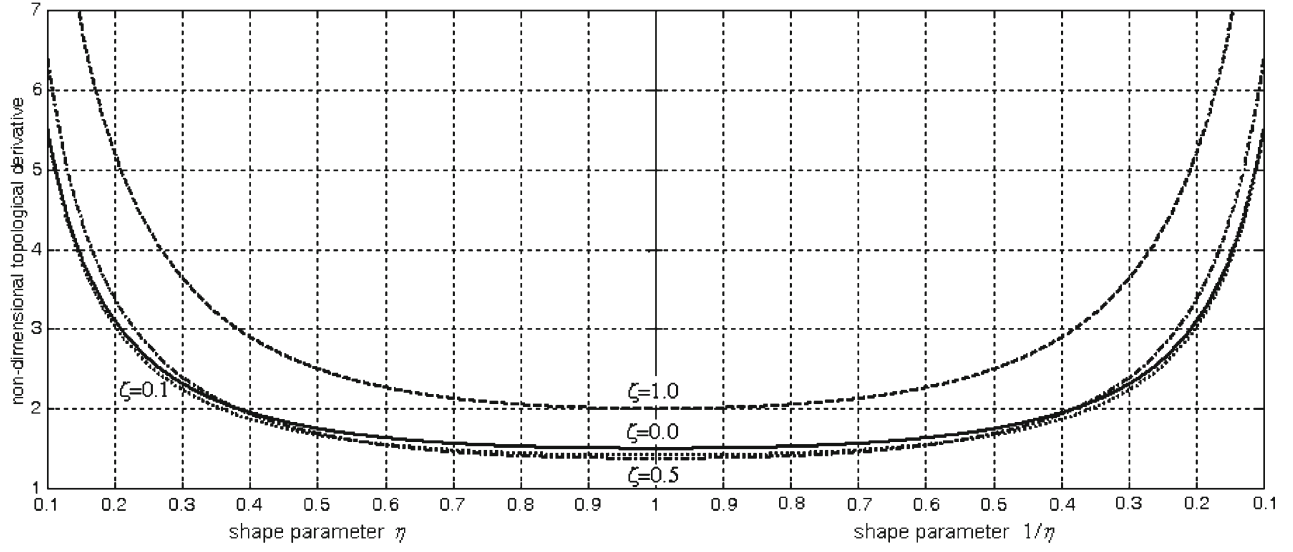


acting on the displacement controlled boundary $\Gamma_{u}$. From the asymptotic analysis, it is seen that

$\left.\boldsymbol{\sigma}\right|_{\xi=0}=\sigma^{(0)}$ in $A,\left.\quad \mathbf{T}\right|_{\xi=0}=\mathbf{T}^{(0)}$ on $\Gamma_{u}$

where $\left.\boldsymbol{\sigma}\right|_{\xi=0}$ and $\left.\mathbf{T}\right|_{\xi=0}$ denote the stress state and reactions for the plate with elliptic hole for $\xi \rightarrow 0$, and $\sigma^{(0)}, \mathbf{T}^{(0)}$ are the states for a homogeneous plate. Similarly as for the functional (2), the relation (4) holds, where in this case

$G^{*}=\int_{A} H\left(\sigma^{(0)}\right) d A+\int_{\Gamma_{u}} h\left(\mathbf{T}^{(0)}\right) d \Gamma_{u}$.

The adjoint structure now can be defined as follows

$\varepsilon^{a i}=\frac{\partial H}{\partial \boldsymbol{\sigma}}, \quad \mathbf{p}^{\mathrm{a} 0}=\mathbf{0}$ in $A_{\xi}$,

$\mathbf{T}^{a 0}=\mathbf{0}$ on $\Gamma_{T}, \quad \mathbf{u}^{a 0}=-\frac{\partial h}{\partial \mathbf{T}}$ on $\Gamma_{u}$,

where $\varepsilon^{a i}$ are the initial strains. The field of initial strains induces the corresponding field of the total strains in the form $\varepsilon^{a}=\varepsilon^{a i}+\varepsilon^{a r}$, where $\varepsilon^{a r}$ is the field of the elastic strains. Now, in view of (46), the first variation of functional (43) with respect to hole expansion process at the material point $\mathbf{x}$, can be expressed analogously to (6), namely

$$
\begin{aligned}
\delta G= & \int_{A_{\xi}} \varepsilon^{a i} \cdot \delta \boldsymbol{\sigma} d A_{\xi}-\int_{\Gamma_{u}} \mathbf{u}^{a 0} \cdot \delta \mathbf{T} d \Gamma_{u} \\
& +\sum_{k=1}^{2} \int_{\Gamma_{\xi}} H n_{k} \delta \Psi_{k} d \Gamma_{\xi} .
\end{aligned}
$$

So, in view of the virtual work equation

$$
\int_{A_{\xi}} \varepsilon^{a r} \cdot \delta \sigma d A_{\xi}=\int_{A_{\xi}} \sigma^{a r} \cdot \delta \varepsilon d A_{\xi}=0,
$$

and the complementary virtual work equation

$$
\begin{aligned}
\int_{A_{\xi}} \varepsilon^{a} \cdot \delta \boldsymbol{\sigma} d A_{\xi}= & \sum_{k=1}^{2} \int_{\Gamma_{\xi}}\left(\mathbf{p}^{0} \cdot \mathbf{u}^{a}-\boldsymbol{\sigma} \cdot \varepsilon^{a}\right) \\
& \times n_{k} \delta \boldsymbol{\Psi}_{k} d \Gamma_{\xi}+\int_{\Gamma_{u}} \mathbf{u}^{a 0} \cdot \delta \mathbf{T} d \Gamma_{u},
\end{aligned}
$$

the first variation of the functional (43) takes the form

$$
\delta G=\sum_{k=1}^{2} \int_{\Gamma_{\xi}}\left(-\boldsymbol{\sigma} \cdot \varepsilon^{a}+\mathbf{p}^{0} \cdot \mathbf{u}^{a}+H\right) n_{k} \delta \boldsymbol{\Psi}_{k} d \Gamma_{\xi} .
$$

Next, assume the function $H(\sigma)$ in a homogeneous form of stress state of degree $2 p$, thus

$H(\boldsymbol{\sigma})=\left(\boldsymbol{\sigma}^{T} \mathbf{S} \boldsymbol{\sigma}\right)^{p}$,

where $\mathbf{S}$ is expressed by (26). Further, using the approach analogous to the analysis described by (15)-(30) and assuming $p=1$, we get

$$
\begin{aligned}
T_{, A_{0}}^{G}(\mathbf{x}, \alpha)= & \frac{1}{2 \pi E} \int_{0}^{2 \pi} \sigma_{s s} \sigma_{s s}^{a} d \theta \\
& -\frac{1}{2 \pi} \int_{0}^{2 \pi}(l+2 m) \sigma_{s s}^{2} d \theta-\mathbf{p}^{0} \cdot \mathbf{u}^{a} .
\end{aligned}
$$

Accounting for (37), (38), the topological sensitivity derivative (52) can be expressed analytically as follows

$$
\begin{aligned}
& T_{, A_{0}}^{G}(\mathbf{x}, \alpha)=\frac{1}{2 E}[\left(\sigma_{1}+\sigma_{2}\right)\left(\sigma_{1}^{a}+\sigma_{2}^{a}\right)\left(\eta+\frac{1}{\eta}\right) \\
&+\left(\sigma_{1}-\sigma_{2}\right)\left(\sigma_{1}^{a}+\sigma_{2}^{a}\right)\left(\eta-\frac{1}{\eta}\right) \cos 2 \alpha \\
&+\left(\sigma_{1}+\sigma_{2}\right)\left(\sigma_{1}^{a}-\sigma_{2}^{a}\right)\left(\eta-\frac{1}{\eta}\right) \\
& \times \cos 2(\alpha-\beta)+\left(\sigma_{1}-\sigma_{2}\right) \\
&\left.\times\left(\sigma_{1}^{a}-\sigma_{2}^{a}\right)\left(\eta+2+\frac{1}{\eta}\right) \cos 2 \beta\right] \\
&-\frac{l+2 m}{2}\left[\left(\sigma_{1}+\sigma_{2}\right)^{2}\left(\eta+\frac{1}{\eta}\right)\right.+2\left(\sigma_{1}^{2}-\sigma_{2}^{2}\right)\left(\eta-\frac{1}{\eta}\right) \\
& \times \cos 2 \alpha+\left(\sigma_{1}-\sigma_{2}\right)^{2} \\
&\left.\times\left(\eta+2+\frac{1}{\eta}\right)\right] \\
&-\mathbf{p}^{0} \cdot \mathbf{u}^{a} . \quad
\end{aligned}
$$

2.4 Sensitivity derivative for the stress energy functional

The particular case occurs when $h(\mathbf{T})=0$ on $\Gamma_{u}, \mathbf{p}^{0}=\mathbf{0}$ at the point $\mathbf{x}$ and the integrand function of (43) is the specific 
stress energy function, $H(\boldsymbol{\sigma})=W(\boldsymbol{\sigma})$. In this case the selfadjoint sensitivity problem is obtained and then

$\sigma_{s s}=\sigma_{s s}^{a}, \quad l=-\frac{v}{2 E}, \quad m=\frac{1+v}{4 E}=\frac{1}{8 G}$.

The topological sensitivity derivative with respect to hole area now has the form

$$
\begin{aligned}
T_{, A_{0}}^{W}(\mathbf{x}, \alpha)=\frac{1}{4 E}[ & \left(\sigma_{1}+\sigma_{2}\right)^{2}\left(\eta+\frac{1}{\eta}\right)+2\left(\sigma_{1}^{2}-\sigma_{2}^{2}\right) \\
& \times\left(\eta-\frac{1}{\eta}\right) \cos 2 \alpha+\left(\sigma_{1}-\sigma_{2}\right)^{2} \\
& \left.\times\left(\eta+2+\frac{1}{\eta}\right)\right]
\end{aligned}
$$

which is identical to (41).

\section{Shape sensitivity derivative and its relation to topological derivative}

In this section the sensitivity analysis with respect to shape variation and principal axes orientation of the ellipse will first be discussed. Next, it will be demonstrated that the topological derivative is obtained from the general sensitivity expression assuming hole growth at fixed hole shape and orientation. Also, the application of shape sensitivity to optimal design of cutouts will be briefly discussed.

\subsection{Sensitivity analysis with respect to semi-axes lengths}

At first, consider the shape variation of the elliptical hole corresponding to length variation of the semi-axis $a$. The shape transformation function (18) and its variation for $\xi=$ 1 now are

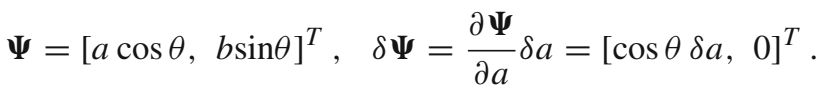

The unit normal vector $\mathbf{n}$ to the hole perimeter is specified by (16) and the length element of boundary $\Gamma_{\xi}$ equals

$d \Gamma_{\xi}=\sqrt{d x_{10}^{2}+d x_{20}^{2}}=\sqrt{a^{2} \sin ^{2} \theta+b^{2} \cos ^{2} \theta} d \theta$.
The variation (14) of the functional $G$ now takes the form

$$
\begin{aligned}
\delta G= & \int_{0}^{2 \pi}\left(-\sigma \cdot \varepsilon^{a}+\mathbf{p}^{0} \cdot \mathbf{u}^{a}+F+f\right) \\
& \times\left(-\frac{b \cos ^{2} \theta}{\sqrt{a^{2} \sin ^{2} \theta+b^{2} \cos ^{2} \theta}} \delta a\right) \\
& \times \sqrt{a^{2} \sin ^{2} \theta+b^{2} \cos ^{2} \theta} d \theta \\
= & b \int_{0}^{2 \pi}\left(\sigma \cdot \varepsilon^{a}-\mathbf{p}^{0} \cdot \mathbf{u}^{a}-F-f\right) \cos ^{2} \theta d \theta \delta a .
\end{aligned}
$$

Assuming the uniform stress states in the hole vicinity of primary and adjoint plates with the principal stresses $\sigma_{1} \geq$ $\sigma_{2}$ and $\sigma_{1}^{a} \geq \sigma_{2}^{a}$, and the integrand function $F(\varepsilon)$ of the form (25) to be quadratic, $p=1$, the integrals of (58) can be analytically expressed after substituting stress states (34) and (36), namely

$$
\begin{aligned}
& b \int_{0}^{2 \pi} \sigma \cdot \varepsilon^{a} \cos ^{2} \theta d \theta \\
&=\frac{b}{E} \int_{0}^{2 \pi} \sigma_{s s} \sigma_{s s}^{a} \cos ^{2} \theta d \theta \\
&=\frac{\pi b}{E}\left[\left(\sigma_{1}+\sigma_{2}\right)\left(\sigma_{1}^{a}+\sigma_{2}^{a}\right) \frac{1}{\eta}-\left(\sigma_{1}-\sigma_{2}\right)\right. \\
& \quad \times\left(\sigma_{1}^{a}+\sigma_{2}^{a}\right) \frac{1}{\eta} \cos 2 \alpha-\left(\sigma_{1}+\sigma_{2}\right)\left(\sigma_{1}^{a}-\sigma_{2}^{a}\right) \\
& \quad \times \frac{1}{\eta} \cos 2(\alpha-\beta)+\left(\sigma_{1}-\sigma_{2}\right) \\
&\left.\quad \times\left(\sigma_{1}^{a}-\sigma_{2}^{a}\right)\left(1+\frac{1}{\eta}\right) \cos 2 \beta\right]
\end{aligned}
$$

and

$$
\begin{aligned}
& b \int_{0}^{2 \pi} F(\varepsilon) \cos ^{2} \theta d \theta \\
& =\frac{b}{E^{2}}\left[\left(1+v^{2}\right)(l+2 m)-2 v l\right] \\
& \quad \times \int_{0}^{2 \pi} \sigma_{s s}^{2} d \theta=\frac{\pi b}{E^{2}}\left[\left(1+v^{2}\right)(l+2 m)-2 v l\right] \\
& \times\left[\left(\sigma_{1}+\sigma_{2}\right)^{2} \frac{1}{\eta}-2\left(\sigma_{1}^{2}-\sigma_{2}^{2}\right) \frac{1}{\eta} \cos 2 \alpha\right. \\
& \left.\quad+\left(\sigma_{1}-\sigma_{2}\right)^{2}\left(1+\frac{1}{\eta}\right)\right] .
\end{aligned}
$$


The variation of $G$ specified by (58) can now be analytically expressed as follows

$$
\begin{aligned}
\delta G=\frac{\partial G}{\partial a} \delta a & \\
=\frac{\pi b}{E}[ & \left(\sigma_{1}+\sigma_{2}\right)\left(\sigma_{1}^{a}+\sigma_{2}^{a}\right) \frac{1}{\eta} \\
& -\left(\sigma_{1}-\sigma_{2}\right)\left(\sigma_{1}^{a}+\sigma_{2}^{a}\right) \frac{1}{\eta} \cos 2 \alpha \\
& -\left(\sigma_{1}+\sigma_{2}\right)\left(\sigma_{1}^{a}-\sigma_{2}^{a}\right) \frac{1}{\eta} \cos 2(\alpha-\beta) \\
& \left.+\left(\sigma_{1}-\sigma_{2}\right)\left(\sigma_{1}^{a}-\sigma_{2}^{a}\right)\left(1+\frac{1}{\eta}\right) \cos 2 \beta\right] \delta a \\
& -\frac{\pi b}{E^{2}}\left[\left(1+v^{2}\right)(l+2 m)-2 v l\right] \\
\times & {\left[\left(\sigma_{1}+\sigma_{2}\right)^{2} \frac{1}{\eta}-2\left(\sigma_{1}^{2}-\sigma_{2}^{2}\right) \frac{1}{\eta} \cos 2 \alpha\right.} \\
& \left.+\left(\sigma_{1}-\sigma_{2}\right)^{2}\left(1+\frac{1}{\eta}\right)\right] \delta a \\
& \int_{0}^{2 \pi}\left(\mathbf{p}^{0} \cdot \mathbf{u}^{a}+f\right) \cos ^{2} \theta d \theta \delta a
\end{aligned}
$$

The self-adjoint case occurs when $F(\varepsilon)=U(\varepsilon)$, where $U(\varepsilon)$ is the specific strain energy. Assuming that $f(\mathbf{u})=$ $0, g(\mathbf{u})=0$ and the body forces $\mathbf{p}^{0}=\mathbf{0}$ in neighborhood of the elliptical hole, we have $\sigma_{s s}=\sigma_{s s}^{a}$ and the functional variation or the sensitivity derivative is

$$
\begin{aligned}
\delta U= & \frac{\partial U}{\partial a} \delta a \\
= & \frac{\pi b}{2 E}\left[\left(\sigma_{1}+\sigma_{2}\right)^{2} \frac{1}{\eta}-2\left(\sigma_{1}^{2}-\sigma_{2}^{2}\right)\right. \\
& \left.\times \frac{1}{\eta} \cos 2 \alpha+\left(\sigma_{1}-\sigma_{2}\right)^{2}\left(1+\frac{1}{\eta}\right)\right] \delta a .
\end{aligned}
$$

It is seen that the sensitivity derivative with respect to $a$ does not vanish for finite lengths values of ellipse semi-axes. For the specified ellipse shape, $\eta=$ const, it is a linear function of the size parameter $a$ and $b$.

Next, consider the shape variation of the elliptical hole corresponding to length variation of the semi-axis $b$. In this case, the shape transformation function (18) and its variation for $\xi=1$ now are

$\boldsymbol{\Psi}=\left[\begin{array}{ll}a \cos \theta, & b \sin \theta\end{array}\right]^{T}, \quad \delta \boldsymbol{\Psi}=\frac{\partial \boldsymbol{\Psi}}{\partial b} \delta b=\left[\begin{array}{ll}0, \sin \theta & \delta b\end{array}\right]^{T}$, and variation (14) of the functional $G$ takes the form

$\delta G=a \int_{0}^{2 \pi}\left(\sigma \cdot \varepsilon^{a}-\mathbf{p}^{0} \cdot \mathbf{u}^{a}-F-f\right) \sin ^{2} \theta d \theta \delta b$.

Using the same approach and the same assumptions as in the case of variation of $a$, finally the variation of $G$ specified by (64) can now be analytically expressed as follows

$$
\begin{aligned}
\delta G=\frac{\partial G}{\partial b} \delta b & \\
=\frac{\pi a}{E} & {\left[\left(\sigma_{1}+\sigma_{2}\right)\left(\sigma_{1}^{a}+\sigma_{2}^{a}\right) \eta+\left(\sigma_{1}-\sigma_{2}\right)\right.} \\
& \times\left(\sigma_{1}^{a}+\sigma_{2}^{a}\right) \eta \cos 2 \alpha+\left(\sigma_{1}+\sigma_{2}\right) \\
& \times\left(\sigma_{1}^{a}-\sigma_{2}^{a}\right) \eta \cos 2(\alpha-\beta)+\left(\sigma_{1}-\sigma_{2}\right) \\
& \left.\times\left(\sigma_{1}^{a}-\sigma_{2}^{a}\right)(1+\eta) \cos 2 \beta\right] \delta b \\
& \quad-\frac{\pi a}{E^{2}}\left[\left(1+v^{2}\right)(l+2 m)-2 v l\right] \\
\times & {\left[\left(\sigma_{1}+\sigma_{2}\right)^{2} \eta+2\left(\sigma_{1}^{2}-\sigma_{2}^{2}\right) \eta \cos 2 \alpha\right.} \\
& \left.+\left(\sigma_{1}-\sigma_{2}\right)^{2}(1+\eta)\right] \delta b \\
& \quad \int_{0}^{2 \pi}\left(\mathbf{p}^{0} \cdot \mathbf{u}^{a}+f\right) \sin ^{2} \theta d \theta \delta b .
\end{aligned}
$$

When the functional $G$ represents the global strain energy and $f(\mathbf{u})=0, g(\mathbf{u})=0, \mathbf{p}^{0}=\mathbf{0}$ in neighborhood of the elliptical hole, the problem becomes self-adjoint and the sensitivity derivative is

$$
\begin{aligned}
& \delta U= \frac{\partial U}{\partial b} \delta b \\
&=\frac{\pi a}{2 E}\left[\left(\sigma_{1}+\sigma_{2}\right)^{2} \eta+2\left(\sigma_{1}^{2}-\sigma_{2}^{2}\right) \eta \cos 2 \alpha\right. \\
&\left.\quad+\left(\sigma_{1}-\sigma_{2}\right)^{2}(1+\eta)\right] \delta b .
\end{aligned}
$$

\subsection{Sensitivity analysis with respect to orientation} of ellipse semi-axes

Consider now the case of rotation of the elliptical hole with respect to the principal stress axes, specified by the varying angle $\alpha$, Fig. 1. The reference system $x_{1}, x_{2}$ follows the principal stress axes and the system $x_{10}, x_{20}$ follows the ellipse axis. Assuming $\xi=1$, the hole boundary point coordinates in the local system $x_{10}, x_{20}$ are

$x_{10}=a \cos \theta, \quad x_{20}=b \sin \theta$, 
and in the global system $x_{1}, x_{2}$ these coordinates are

$$
x_{1}=x_{10} \cos \alpha-x_{20} \sin \alpha=a \cos \theta \cos \alpha-b \sin \theta \sin \alpha,
$$$$
x_{2}=x_{10} \sin \alpha+x_{20} \cos \alpha=a \cos \theta \sin \alpha+b \sin \theta \cos \alpha \text {. }
$$

The hole boundary transformation vector due to rotation is now specified as follows

$$
\begin{array}{r}
\delta \boldsymbol{\Psi}=\frac{\partial \boldsymbol{\Psi}}{\partial \alpha} \delta \alpha=[-(a \cos \theta \sin \alpha+b \sin \theta \cos \alpha) \delta \alpha, \\
(a \cos \theta \cos \alpha-b \sin \theta \sin \alpha) \delta \alpha]^{T}
\end{array}
$$

and the unit normal vector and length element are

$\mathbf{n}=\left[-\frac{d x_{2}}{\sqrt{d x_{1}^{2}+d x_{2}^{2}}}, \frac{d x_{1}}{\sqrt{d x_{1}^{2}+d x_{2}^{2}}}\right]^{T}, d \Gamma_{\xi}=\sqrt{d x_{1}^{2}+d x_{2}^{2}}$.

In view of (69), (70), the variation (10) of the functional is now expressed as follows

$$
\begin{aligned}
\delta G= & \frac{1}{2} a b\left(\eta-\frac{1}{\eta}\right) \\
& \times \int_{0}^{2 \pi}\left(-\sigma \cdot \varepsilon^{a}+\mathbf{p}^{0} \cdot \mathbf{u}^{a}+F+f\right) \sin 2 \theta d \theta \delta \alpha
\end{aligned}
$$

and for the quadratic function $F(\varepsilon)$ of the form (25), $p=1$, we obtain assuming the uniformly loaded plate

$$
\begin{aligned}
\delta G= & \frac{\partial G}{\partial \alpha} \delta \alpha \\
= & -\frac{A}{E}\left(\eta-\frac{1}{\eta}\right)\left\{\left(\sigma_{1}-\sigma_{2}\right)\left(\sigma_{1}^{a}+\sigma_{2}^{a}\right) \sin 2 \alpha\right. \\
& +\left(\sigma_{1}+\sigma_{2}\right)\left(\sigma_{1}^{a}-\sigma_{2}^{a}\right) \sin 2(\alpha-\beta) \\
& \left.-\frac{2}{E}\left[\left(1+v^{2}\right)(l+2 m)-2 v l\right]\left(\sigma_{1}^{2}-\sigma_{2}^{2}\right) \sin 2 \alpha\right\} \delta \alpha \\
& +\frac{1}{2} a b\left(\eta-\frac{1}{\eta}\right) \int_{0}^{2 \pi}\left(\mathbf{p}^{0} \cdot \mathbf{u}^{a}+f\right) \sin 2 \theta d \theta \delta \alpha,
\end{aligned}
$$

where $A=\pi a b$. For the particular case, when $F(\varepsilon)=$ $U(\varepsilon)$, and $f(\mathbf{u})=0, g(\mathbf{u})=0, \mathbf{p}^{0}=\mathbf{0}$ in neighborhood of the elliptical hole, the self-adjoint problem occurs. Then, we have

$$
\delta U=\frac{\partial U}{\partial \alpha} \delta \alpha=-\frac{A}{E}\left(\eta-\frac{1}{\eta}\right)\left(\sigma_{1}^{2}-\sigma_{2}^{2}\right) \sin 2 \alpha \delta \alpha .
$$

\subsection{Shape sensitivity analysis for constant hole area}

Consider the shape variation of the elliptical hole by assuming constant area $A=\pi a b=$ const. For varying lengths of semi-axes $a$ and $b$ the area constraint provides the relation

$\delta b=-\frac{b}{a} \delta a$.

Then, the analyzed shape sensitivity derivative takes the form

$\delta G=\frac{\partial G}{\partial a} \delta a+\frac{\partial G}{\partial b} \delta b=\left(\frac{\partial G}{\partial a}-\frac{b}{a} \frac{\partial G}{\partial b}\right) \delta a$

and, in view of (61) and (65), it can be expressed as follows

$$
\begin{aligned}
\delta G=-\frac{\pi b}{E}[ & \left(\sigma_{1}+\sigma_{2}\right)\left(\sigma_{1}^{a}+\sigma_{2}^{a}\right)\left(\eta-\frac{1}{\eta}\right) \\
& +\left(\sigma_{1}-\sigma_{2}\right)\left(\sigma_{1}^{a}+\sigma_{2}^{a}\right)\left(\eta+\frac{1}{\eta}\right) \cos 2 \alpha \\
& +\left(\sigma_{1}+\sigma_{2}\right)\left(\sigma_{1}^{a}-\sigma_{2}^{a}\right)\left(\eta+\frac{1}{\eta}\right) \\
& \times \cos 2(\alpha-\beta)+\left(\sigma_{1}-\sigma_{2}\right)\left(\sigma_{1}^{a}-\sigma_{2}^{a}\right) \\
& \left.\times\left(\eta+\frac{1}{\eta}\right) \cos 2 \beta\right] \delta a \\
+ & \frac{\pi b}{E^{2}}\left[\left(1+v^{2}\right)(l+2 m)-2 v l\right] \\
\times & \left(\sigma_{1}+\sigma_{2}\right)^{2}\left(\eta-\frac{1}{\eta}\right)-2\left(\sigma_{1}^{2}-\sigma_{2}^{2}\right) \\
& \left.\times\left(\eta+\frac{1}{\eta}\right) \cos 2 \alpha+\left(\sigma_{1}-\sigma_{2}\right)^{2}\left(\eta-\frac{1}{\eta}\right)\right] \delta a \\
& \quad b \int_{0}^{2 \pi}\left(\mathbf{p}^{0} \cdot \mathbf{u}^{a}+f\right) \cos 2 \theta d \theta \delta a .
\end{aligned}
$$

For the particular case, when the functional $G$ represents the global strain energy and $f(\mathbf{u})=0, g(\mathbf{u})=0, \mathbf{p}^{0}=\mathbf{0}$ in neighborhood of the elliptical hole, the problem becomes self-adjoint and the shape sensitivity is

$$
\begin{aligned}
\delta U=\frac{\partial U}{\partial a} \delta a=-\frac{\pi b}{2 E}[ & \left(\sigma_{1}+\sigma_{2}\right)^{2}\left(\eta-\frac{1}{\eta}\right) \\
& +2\left(\sigma_{1}^{2}-\sigma_{2}^{2}\right)\left(\eta+\frac{1}{\eta}\right) \cos 2 \alpha \\
& \left.+\left(\sigma_{1}-\sigma_{2}\right)^{2}\left(\eta-\frac{1}{\eta}\right)\right] \delta a .
\end{aligned}
$$


3.4 Topological derivative as the size sensitivity analysis problem

Consider the problem of determination of the topological derivative with respect to introduction of the infinitesimally small elliptic hole. We have the size growth relations

$a^{(\bmod )}=\xi a, \quad b^{(\bmod )}=\xi b$,

where $a^{\text {(mod) }}, b^{\text {(mod) }}$ are the semi-axes lengths controlled by the expansion parameter $\xi$. The topological derivative can be calculated as the derivative with respect to the expansion parameter $\xi$ for $\xi=0$. Thus, in view of (22), we have

$$
\begin{aligned}
T_{, A_{0}}^{G}(\mathbf{x}, \alpha)= & \left.\left(\frac{\partial G}{\partial \xi} \frac{\partial \xi}{\partial A}\right)\right|_{\xi=0} \\
= & {\left[\left(\frac{\partial G}{\partial a^{(\bmod )}} \frac{\partial a^{(\bmod )}}{\partial \xi}\right.\right.} \\
& \left.\left.+\frac{\partial G}{\partial} b^{(\bmod )} \frac{\partial b^{(\bmod )}}{\partial \xi}\right) \frac{\partial \xi}{\partial A}\right]\left.\right|_{\xi=0} .
\end{aligned}
$$

Taking into account, that $\partial G / \partial a^{(\bmod )}, \partial G / \partial b^{(\bmod )}$ are specified by (61) and (65), and

$\frac{\partial a^{(\mathrm{mod})}}{\partial \xi}=a, \quad \frac{\partial b^{(\mathrm{mod})}}{\partial \xi}=b, \quad \frac{\partial \xi}{\partial A}=\frac{1}{2 \pi \xi a b}$,

the topological derivative is finally expressed by the same formula, namely by (39), as previously.

\subsection{Optimization of shape and orientation of elliptical holes}

Consider a general optimization problem defined as follows

$\min _{p_{i}, i=1,2, \ldots, n} G, \quad$ subject to $C-C_{0} \leq 0$,

where $G\left(p_{i}\right)$ is the objective functional (2), $C\left(p_{i}\right)$ denotes the global cost and $C_{0}$ is the upper bound on the global cost. Introducing the Lagrangian functional

$$
L\left(p_{i}, \lambda\right)=G+\lambda\left(C-C_{0}\right), \quad \lambda \geq 0
$$

the stationary conditions are

$$
\begin{aligned}
& \frac{\partial G}{\partial p_{i}}+\lambda \frac{\partial C}{\partial p_{i}}=0, \quad i=1,2, \ldots, n, \\
& \lambda\left(C-C_{0}\right)=0
\end{aligned}
$$

where $\lambda$ is the Lagrange multiplier and $p_{i}, i=1,2, \ldots n$ are the design parameters. The sensitivity derivatives $\partial G / \partial p_{i}$ are specified by the formulae derived in the paper, where the size parameter $\xi$, shape parameters $a$ and $b$, or orientation parameter $\alpha$ are to be determined. The cost derivatives $\partial C / \partial p_{i}$ can easily be derived for the assumed cost function expressed in terms of design parameters. The optimal values of the parameters $p_{i}$ and the multiplier $\lambda$ are determined in the incremental process of gradient optimization.

\subsection{Optimal determination of shape and orientation of elliptical hole of constant area}

Consider a simple problem when the plate is subjected to the uniform remote principal stresses $\sigma_{1}, \sigma_{2}\left(\left|\sigma_{1}\right| \geq\left|\sigma_{2}\right|\right)$, following the $x_{1}, x_{2}$ coordinate axes, Fig. 5a. The optimal design problem is aimed to specify lengths of semi-axes $a, b$, and orientation angle $\alpha$ of the hole assuming its area $A_{0}=\pi a b$ to be fixed. The optimization problem is to maximize global plate stiffness expressed by the potential energy $\Pi$, or to minimize global compliance measured by the strain energy, thus

$$
\begin{aligned}
& \max _{a, b, \alpha}\left[\int_{A_{\xi}} \Pi(\varepsilon, \mathbf{u}) d A_{\xi}\right] \text { or } \min _{a, b, \alpha}\left[\int_{A_{\xi}} U(\varepsilon) d A_{\xi}\right], \\
& \text { subject to } \pi a b=A_{0} .
\end{aligned}
$$

Using the sensitivity derivatives (77) and (73), the stationary conditions can easily be stated in the form

$$
\begin{aligned}
& \left(\sigma_{1}+\sigma_{2}\right)^{2}\left(\eta-\frac{1}{\eta}\right)+2\left(\sigma_{1}^{2}-\sigma_{2}^{2}\right)\left(\eta+\frac{1}{\eta}\right) \cos 2 \alpha \\
& +\left(\sigma_{1}-\sigma_{2}\right)^{2}\left(\eta-\frac{1}{\eta}\right)=0 \\
& \left(\eta-\frac{1}{\eta}\right)\left(\sigma_{1}^{2}-\sigma_{2}^{2}\right) \sin 2 \alpha=0 .
\end{aligned}
$$

For $\eta \neq 1$ and $\sigma_{1} \neq \sigma_{2}$ the optimal orientation of ellipse axes is $\alpha=0$ or $\alpha=\pi / 2$. The maximal stiffness design is obtained by setting

$\alpha=0, \quad \frac{b}{a}=\eta=\frac{\left|\sigma_{2}\right|}{\left|\sigma_{1}\right|}$

and the maximal compliance design corresponds to the parameters

$\alpha=\frac{\pi}{2}, \quad \frac{b}{a}=\eta=\frac{\left|\sigma_{2}\right|}{\left|\sigma_{1}\right|}$ 
Fig. 5 Elliptical hole in a plate uniformly loaded on its boundaries

$x_{1}= \pm L_{1} / 2, x_{1}= \pm L_{1} / 2$ :

$x_{2}= \pm L_{2} / 2, x_{2}= \pm L_{2} / 2$ :

a) design parameters; b) optimal position of the hole for maximal stiffness design; c) optimal position of the hole for maximal compliance design a)

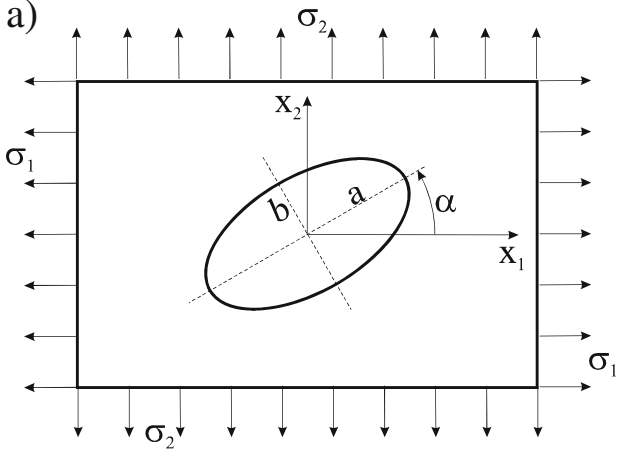

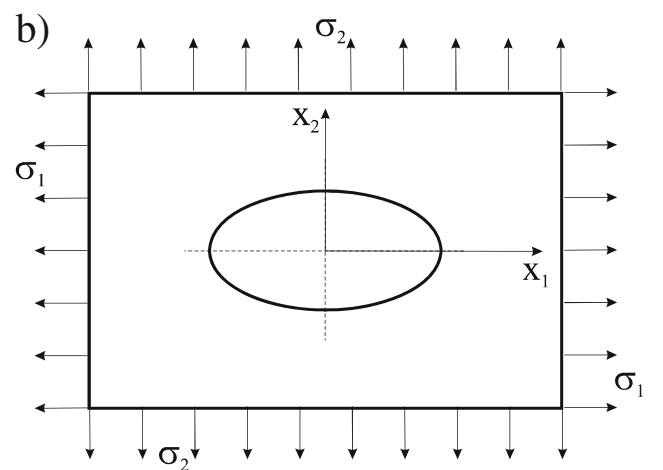

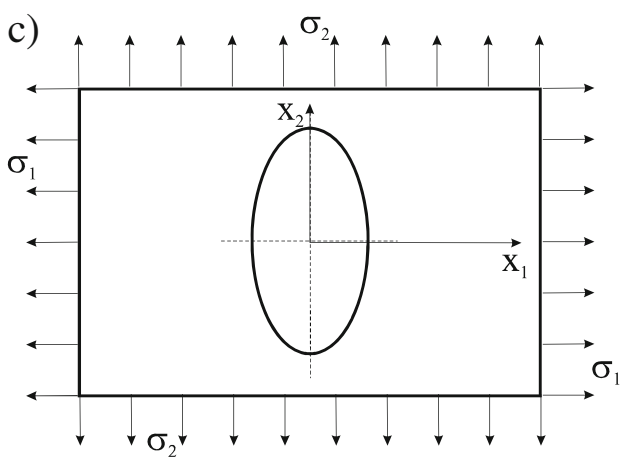

The optimal designs are shown in Fig. 5b, c. It is seen that the optimal orientation of the ellipse corresponds to coaxiality of ellipse and principal stress axes.

\subsection{Localization of maximal stresses and crack orientation}

In order to find location of crack initiation on the boundary of the elliptic hole, the maximal stress criterion can be used. The distribution of the stresses on the rim of the hole is expressed by (34). Position of the maximal stress $\sigma_{s s}$ determined by the angle $\theta$ (Fig. 2) in function of stress ratio $\sigma_{2} / \sigma_{1}$ for selected values of the shape parameter $\eta$ and for the orientation angles $\alpha=\pi / 6, \alpha=\pi / 4, \alpha=\pi / 3$, $\alpha=-\pi / 4$ is presented respectively in Fig. $6 \mathrm{a}, \mathrm{b}, \mathrm{c}, \mathrm{d}$.

Let us note that in order to specify position of a point on the ellipse boundary, instead of the angle $\theta$ also angles $\beta$ and $\gamma$ can be used (Fig. 2), where the relations between these angles are as follows

$\tan \gamma=\frac{\tan \theta}{\eta}, \quad \tan \beta=\eta \tan \theta$

and $\gamma$ specifies the orientation of crack growth in normal direction to the hole perimeter.

\subsection{Transition to singular case: sensitivity analysis for plane crack}

Consider now the case of a plane crack of length $2 a$ in a uniformly loaded infinite plate, inclined at the angle $\alpha$ to the major tensile stress $\sigma_{1}$ of the primary state (Fig. 7) and at the angle $\alpha-\beta$ to the major stress $\sigma_{1}^{a}$ of the adjoint state.

The crack can be treated as the limiting case of the elliptical hole by setting $b=0,1 / \eta=\infty$ in the derived formulae. The sensitivity derivative with respect to the hole area is now infinite but the derivative with respect to length of ellipse semi-axis is finite and depends linearly on the crack length $2 a$. Consider first the self-adjoint case for which the sensitivity derivative of the strain plate energy $U=U(\mathbf{u}, a)$, or the potential plate energy $\Pi=\Pi(\mathbf{u}, a)$, is specified. From (62) for $b=0,1 / \eta=\infty$ it follows that

$G_{c}=-\frac{\delta \Pi}{\delta a}=\frac{\partial U}{\partial a}=\frac{\pi a}{E}\left[\sigma_{1}^{2}+\sigma_{2}^{2}-\left(\sigma_{1}^{2}-\sigma_{2}^{2}\right) \cos 2 \alpha\right]$.

For $\alpha=0$ the crack is oriented along the $\sigma_{1}$ - axis and then we have

$G_{c}=\frac{2 \pi a \sigma_{2}^{2}}{E}=\frac{2 K_{I}^{2}}{E}, \quad K_{I}=\sigma_{2} \sqrt{\pi a}$.

Similarly, for $\alpha=\pi / 4$ and $\sigma_{1}=-\sigma_{2}=\sigma_{12}$, the plane crack is subjected to shear stress and there is

$G_{c}=\frac{2 \pi a \sigma_{12}^{2}}{E}=\frac{2 K_{I I}^{2}}{E}, \quad K_{I I}=\sigma_{12} \sqrt{\pi a}$. 
a)

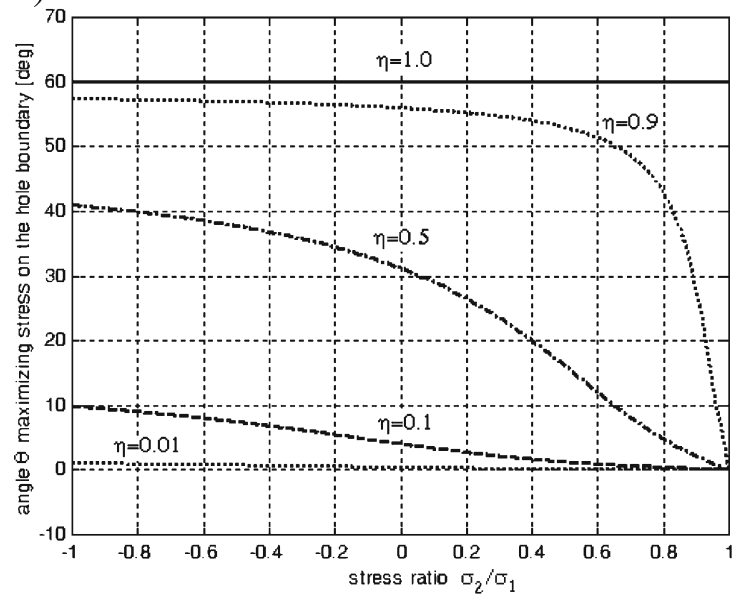

c)

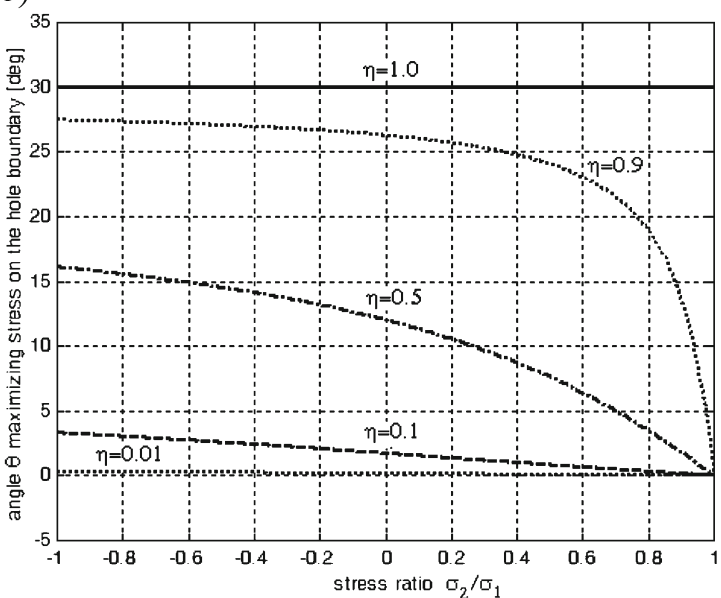

b)

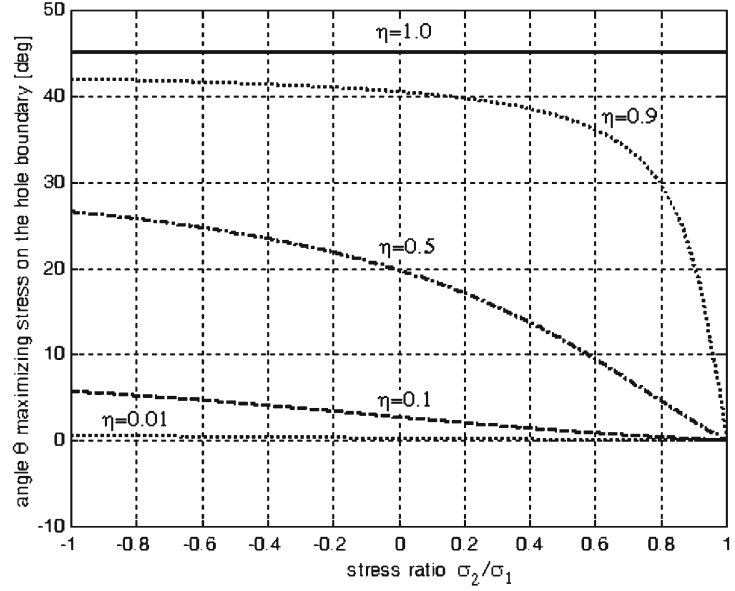

d)

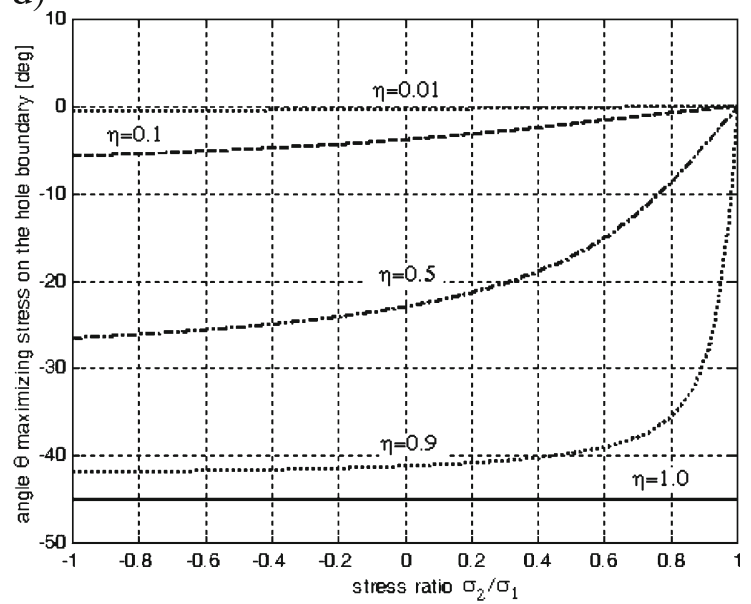

Fig. 6 Localization of the maximal stress $\sigma_{s s}$ on the hole boundary specified by the angle $\theta$ in function of stress ratio $\sigma_{1} / \sigma_{2}$ for orientation angles: a) $\alpha=\pi / 6$; b) $\alpha=\pi / 4$; c) $\alpha=\pi / 3$; d) $\alpha=-\pi / 4$

Here $K_{I}$ and $K_{I I}$ denote the stress intensity factors of tension and shear modes specifying the singular asymptotic stress fields at the crack tip expressed in the local polar coordinate system $(r, \theta)$, so

$\sigma_{i j}^{I}=\frac{K_{I}}{\sqrt{2 \pi r}} f_{i j}^{I}(\theta), \quad \sigma_{i j}^{I I}=\frac{K_{I I}}{\sqrt{2 \pi r}} f_{i j}^{I I}(\theta)$.

Referring the stress field to the coordinate axes $x_{10}, x_{20}$ coaxial and normal to the crack, Fig. 7b, in view of the stress transformation formulae, the sensitivity derivative (89) takes the form well familiar in fracture mechanics (cf. Miannay 1998), thus

$G_{c}=\frac{2 \pi a}{E}\left(\sigma_{22}^{2}+\sigma_{12}^{2}\right)=2\left(\frac{K_{I}^{2}}{E}+\frac{K_{I I}^{2}}{E}\right)$ and it expresses the release of potential energy due to crack growth.

In the general case of an arbitrary functional $G$, its sensitivity in view of (61), assuming $f(\mathbf{u})=0, \mathbf{p}^{0}=\mathbf{0}$, is expressed as follows

$$
\begin{aligned}
\frac{\partial G}{\partial a}= & \frac{\pi a}{E}\left[\left(\sigma_{1}+\sigma_{2}\right)\left(\sigma_{1}^{a}+\sigma_{2}^{a}\right)-\left(\sigma_{1}-\sigma_{2}\right)\right. \\
& \times\left(\sigma_{1}^{a}+\sigma_{2}^{a}\right) \cos 2 \alpha-\left(\sigma_{1}+\sigma_{2}\right)\left(\sigma_{1}^{a}-\sigma_{2}^{a}\right) \\
& \left.\times \cos 2(\alpha-\beta)+\left(\sigma_{1}-\sigma_{2}\right)\left(\sigma_{1}^{a}-\sigma_{2}^{a}\right) \cos 2 \beta\right] \\
& -\frac{2 \pi a}{E^{2}}\left[\left(1+v^{2}\right)(l+2 m)-2 v l\right] \\
& \times\left[\sigma_{1}^{2}+\sigma_{2}^{2}-\left(\sigma_{1}^{2}-\sigma_{2}^{2}\right) \cos 2 \alpha\right]
\end{aligned}
$$


Fig. 7 Crack in the plate: a) inclined with respect to principal stress axes, b) in the coordinates $x_{10}, x_{20}$ referred to the crack plane

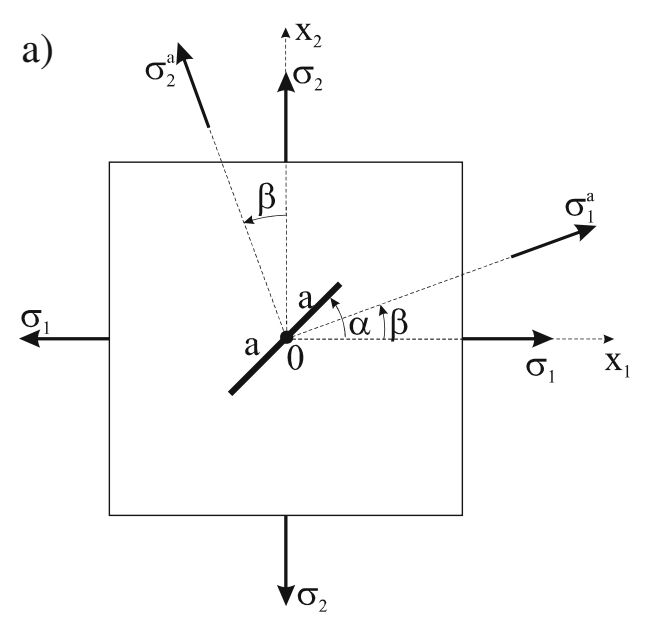

Referring to the coordinate system $x_{10}, x_{20}$ the sensitivity expression (94) can be presented in the form

$$
\begin{aligned}
\frac{\partial G}{\partial a}= & \frac{4 \pi a}{E}\left(\sigma_{22} \sigma_{22}^{a}+\sigma_{12} \sigma_{12}^{a}\right)-\frac{4 \pi a}{E^{2}} \\
& \times\left[\left(1+v^{2}\right)(l+2 m)-2 v l\right]\left(\sigma_{22}^{2}+\sigma_{12}^{2}\right) \\
= & 4\left(\frac{K_{I} K_{I}^{a}}{E}+\frac{K_{I I} K_{I I}^{a}}{E}\right) \\
& -\frac{4}{E}\left[\left(1+v^{2}\right)(l+2 m)-2 v l\right]\left(\frac{K_{I}^{2}}{E}+\frac{K_{I I}^{2}}{E}\right),
\end{aligned}
$$

where $K_{I}^{a}, K_{I I}^{a}$ are the stress intensity factors associated with the adjoint stress state. The sensitivity derivatives (93) and (95) are linearly related to the crack length $2 a$ and vanish for $a=0$. The Griffith crack generation governed by the critical value of the potential energy release therefore cannot occur. However, the crack generation of finite length can be assumed as physically admissible.

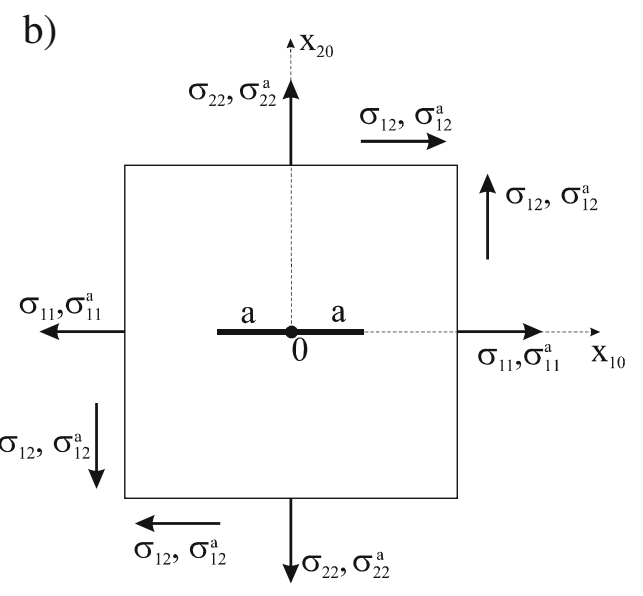

Consider, for instance a plane crack in a wide plate under uniaxial tension, Fig. 8. The presence of crack induces the release of potential energy $\Delta \Pi_{l}$ and growth of surface or dissipated energy $\Delta \Pi_{s}$ thus the total energy variation is

$\Delta \Pi=\Delta \Pi_{l}+\Delta \Pi_{s}=\frac{-\pi a^{2} \sigma^{2}}{E}+2 \gamma a$,

where $\gamma$ is the specific surface energy per unit crack length (cf. Bojczuk and Mróz 2007). The critical crack length is specified from the condition

$\frac{\partial(\Delta \Pi)}{\partial a}=0, \quad$ and $\quad a_{c}=\frac{\gamma E}{\pi \sigma^{2}}$.

Let us note that for $a>a_{c}$ there is unstable crack growth at constant stress with associated decrease of the total energy. The other length is obtained from the constant energy transformation $\Delta \Pi=0$ providing the crack length

$a_{i}=\frac{2 \gamma E}{\pi \sigma^{2}}=2 a_{c}$.
Fig. 8 Plane crack under tensile stress: a) plate element, b) variation of potential and dissipated surface energies a)

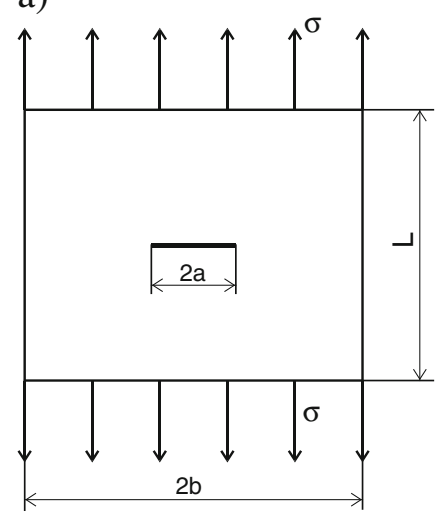

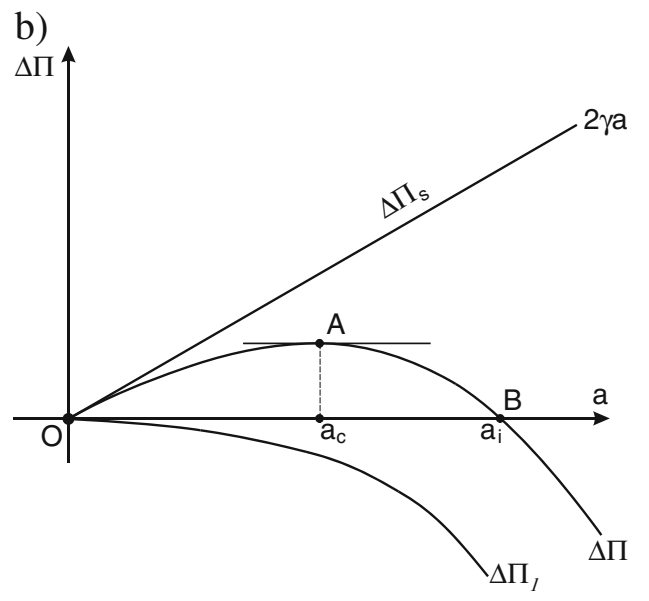


The finite topology transformation then occurs along the path OB, Fig. 8b.

The concept of finite topology variation was discussed by Bojczuk and Mróz $(2003,2009)$ and applied in optimal design of discrete supports in beam structures and of plate structures.

Let us also note that the transition from an ellipse to a crack cannot be always conducted within the regular perturbation theory, as it was shown by Il'in and Gadyl'shin (2001).

\section{Concluding remarks}

The expression of topological derivative with respect to the area of an infinitesimally small elliptical hole and the expressions of sensitivity derivatives with respect to shape and orientation of finite elliptical holes have been derived in the explicit forms in the paper. It was demonstrated that the topological derivative can be obtained from the shape sensitivity analysis with respect to variation of the ellipse axes lengths. It varies with the orientation of the ellipse axes, with respect to the principal stress axes and also with the shape ratio $\eta=b / a$. For $\eta=0$ or $\eta=\infty$, that is for a limiting case of plane crack the topological derivative under biaxial stress becomes infinite but the sensitivity derivative with respect to the crack length is a linear function of length. For arbitrary stress or displacement functionals the topological derivative is expressed in terms of primary and adjoint stress or strain fields of a uniform plate.

The analysis presented can be generalized to the case of sensitivity with respect to introduced spherical or ellipsoidal cavities and inclusions of differing materials. The analytical expression of the topological derivative in these cases would certainly improve the optimal design methodology of structures and composite materials.

Open Access This article is distributed under the terms of the Creative Commons Attribution Noncommercial License which permits any noncommercial use, distribution, and reproduction in any medium, provided the original author(s) and source are credited.

\section{References}

Allaire G, Jouve F, Toader A-M (2004) Structural optimization using sensitivity analysis and a level set method. J Comput Phys 194:363-393

Ammari H, Kang H (2004) Reconstruction of small inhomogeneities from boundary measurements. Lecture Notes in Mathematics 1846. Springer-Verlag, Berlin

Amstutz S (2006) Sensitivity analysis with respect to a local perturbation of the material property. Asymptot Anal 49:87-108

Bendsoe MP, Sigmund O (2003) Topology optimization: theory, methods and applications. Springer, Heilderberg
Bojczuk D, Mróz Z (2003) Finite topology variations in optimal design of structures. Struct Multidisc Optim 25:153-173

Bojczuk D, Mróz Z (2007) Some applications of topological sensitivity derivative. In: Proceedings of the seventh congress of structural and multidisciplinary optimization, pp 1817 1826

Bojczuk D, Mróz Z (2009) Topological sensitivity derivative and finite topology modifications: application to optimization of plates in bending. Struct Multidisc Optim 39:1-15

Bojczuk D, Szteleblak W (2008) Optimization of layout and shape of stiffeners in 2D structures. Comput Struct 86:14361446

Burger M, Hackl B, Ring W (2004) Incorporating topological derivatives into level set method. J Comput Phys 194:344362

Cea JS, Garreau S, Guillaume P, Masmoudi M (2000) The shape and topological optimizations connection. Comput Methods Appl Mech Eng 188:713-726

Dems K, Mróz Z (1984) Variational approach by means of adjoint systems to structural optimization and sensitivity analysis. II. Structure shape variation. Int J Solids Struct 19:527-552

Dems K, Mróz Z (1986) On a class of conservation rules associated with sensitivity analysis in linear elasticity. Int J Solids Struct 22:737-758

Eschenauer HA, Olhoff N (2001) Topology optimization of continuum structures: a review. Appl Mech Rev 54:331-390

Feijóo RA, Padra C, Saliba R, Taroco E, Venere MJ (2000) Shape sensitivity analysis for energy release rate evaluation and its application to the study of three-dimensional cracked bodies. Comput Methods Appl Mech Eng 188:649-664

Gao X-L (1996) A general solution of an infinite elastic plate with an elliptic hole under biaxial loading. Int J Press Vessels Piping 67:95-104

Il'in AM, Gadyl'shin RR (2001) On the limits of applicability of regular perturbation theory for a membrane with a narrow crack (in Russian). Dokl Akad Nauk 380:172-175

Khludnev AM, Novotny AA, Sokołowski J, Żochowski T (2009) Shape and topology sensitivity analysis for cracks in elastic bodies on boundaries of rigid inclusions. J Mech Phys Solids 57:1718 1732

Kienzler R, Fischer FD, Fratzl P (2006) On energy changes due to formation of a circular hole in an elastic plate. Arch Appl Mech 76:681-697

Miannay DP (1998) Fracture mechanics. Springer, Mechanical Engineering Series

Mróz Z, Bojczuk D (2006) Topological sensitivity derivative: application in optimal design and material science. Found Civil Environ Eng 7:229-250

Nazarov SA, Sokołowski J (2003) Asymptotic analysis of shape functionals. J Math Pures Appl 82:125-196

Nazarov SA, Sokołowski J, Specovius-Neugebauer M (2010) Polarization matrices in anisotropic heterogeneous elasticity. Asymptot Anal 68:189-221

Novotny AA, Feijóo RA, Taroco E, Padra C (2003) Topological sensitivity analysis. Comput Methods Appl Mech Eng 192:803829

Rozvany GIN (2009) A critical review of established methods of structural topology optimization. Struct Multidisc Optim 37:319323

Silva M, Matalon M, Tortorelli DA (2010) Higher order topological derivatives in elasticity. Int J Solids Struct 47:30533066

Silva M, Geubelle PH, Tortorelli DA (2011) Energy release rate approximation for small surface-breaking cracks using the topological derivative. J Mech Phys Solids 59:925939 
Sokołowski J, Żochowski T (1999) On topological derivative in shape optimization. SIAM J Control Optim 37:1251-1272

Sokołowski J, Żochowski T (2003) Optimality conditions for simultaneous topology and shape optimization. SIAM J Control Optim 42:1198-1221

Taroco E (2000) Shape sensitivity analysis in linear elastic fracture mechanics. Comput Methods Appl Mech Eng 188:697-712
Timoshenko S, Goodier JN (1951) Theory of elasticity. McGraw-Hill, New York-Toronto-London

Van Goethem N, Novotny AA (2010) Crack nucleation sensitivity analysis. Math Methods Appl Sci 33:1978-1994

Wang X, Wang MY, Guo D (2004) Structural shape and topology optimization in a level-set-based framework of region representation. Struct Multidisc Optim 27:1-19 